\title{
RESPUESTAS DE LOS ATRIBUTOS FOTOSINTÉTICOS Y ESTEQUIOMÉTRICOS A LA ARIDEZ EN LAS ESPECIES Y TIPOS FUNCIONALES DE DOS COMUNIDADES DEL DESIERTO SONORENSE RESPONSES OF PHOTOSYNTHETIC AND STOICHIOMETRIC TRAITS TO ARIDITY IN SPECIES AND FUNCTIONAL TYPES OF TWO SONORAN DESERT PLANT COMMUNITIES
}

\author{
-Delia Marina Acuña-Acosta, @Alejandro E. Castellanos-Villegas*, \\ ¿ José Manuel Llano-Sotelo, and Đ José Raúl Romo-León
}

DICTUS, Universidad de Sonora. Hermosillo, Sonora, México.

*Autor de correspondencia: alejandro.castellanos@unison.mx

\section{Resumen}

Antecedentes: Las plantas de zonas secas han desarrollado adaptaciones para la eficiencia en el uso de agua (UEA), aunque la limitación de nutrientes y exceso de irradiancia también afectan su desempeño. Altas tasas de fotosíntesis y contenido de nitrógeno foliar reportado previamente parecen contradecir la alta UEA en sus especies y comunidades.

Preguntas: ¿Cómo son los atributos foliares ecofisiológicos y estequiométricos en especies desérticas? ¿Favorecen las especies desérticas la mayor eficiencia en el uso del agua y/o nutrientes?

Especies de estudio: Calliandra eriophylla, Cercidium microphyllum, Encelia farinosa, Eysenhardtia orthocarpa, Fouquieria diguetii, Fouquieria macdougalii, Ipomoea arborescens, Jatropha cardiophylla, Jatropha cordata, Larrea tridentata, Mimosa laxiflora, Olneya tesota y Prosopis velutina.

Sitio y años de estudio: Desierto Sonorense, Región central de Sonora, México. Agosto- septiembre de 2019.

Métodos: Se midieron y analizaron atributos ecofisiológicos y estequiométricos foliares en especies y tipos funcionales de dos comunidades. Resultados: Se encontró mayor variabilidad en atributos fotosintéticos y de la fluorescencia que en la composición elemental y cocientes estequiométricos. Las especies en el sitio árido mostraron tendencias de mayores tasas fotosintéticas e integridad fotoquímica y baja UEA. En la comunidad semiárida los atributos foliares se asociaron con mayor composición elemental $\left(\mathrm{N}_{\text {mass, }} \mathrm{P}_{\text {mass }}\right)$, menores cocientes estequiométricos y eficiencia de uso de nitrógeno (EUN).

Conclusiones: Los atributos foliares de las especies y tipos funcionales del Desierto Sonorense se correlacionaron con los mecanismos de fotoprotección y no las eficiencias de uso de recursos instantáneas, privilegiando la ganancia de $\mathrm{C}$ a largo plazo.

Palabras clave: $\mathrm{C}, \mathrm{N}$ y $\mathrm{P}$ foliares, espectro económico de la hoja, estequiometría, fluorescencia, fotosíntesis.

\section{Abstract}

Background: Plants in drylands have developed several water use efficiency (WUE) adaptations, although nutrient limitations and excess irradiances affect also their performance. Previous findings of high photosynthetic rates and nitrogen concentration in leaves, may contradict the known low productivity in their communities.

Questions: How different are leaf ecophysiological and stoichiometric traits in dryland species? Did species favor higher water or nutrient use efficiencies in contrasting dryland communities?

Studied species / data description /Mathematical model: Calliandra eriophylla, Cercidium microphyllum, Encelia farinosa, Eysenhardtia orthocarpa, Fouquieria diguetii, Fouquieria macdougalii, Ipomoea arborescens, Jatropha cardiophylla, Jatropha cordata, Larrea tridentata, Mimosa laxiflora, Olneya tesota and Prosopis velutina.

Study site and dates: Sonoran Desert, Central Sonora Region, Mexico. August - September 2019.

Methods: Ecophysiological and stoichiometric leaf traits were measured and analyzed for dominant species and functional types from two Sonoran Desert plant communities.

Results: We found greater variability in photosynthetic and fluorescence traits than in elemental composition $\left(\mathrm{N}_{\text {mass }}, \mathrm{P}_{\text {mass }}\right)$ and stoichiometry ratios. Species in the arid site tend to have higher photosynthetic rates and photochemical integration but low WUE. In the semiarid community, leaf traits were associated to higher elemental composition $\left(\mathrm{N}_{\text {mass }}, \mathrm{P}_{\text {mass }}\right)$ but low stoichiometric ratios and nitrogen use efficiency (NUE).

Conclusions: Leaf Traits in Sonoran Desert species and functional types correlated with photoprotective mechanisms rather than instantaneous resources use efficiencies, to ensure long term $\mathrm{C}$ gain.

Keywords: Chlorophyll fluorescence, C, N y P in leaves, economic spectrum, photosynthesis, stoichiometry.

Este artículo se encuentra bajo los términos de la licencia Creative Commons Attribution License CCBY-NC (4.0) internacional. https://creativecommons.org/licenses/by-nc/4.0/ 
Las zonas secas (áridas, semiáridas y subhúmedas) cubren más del $40 \%$ de la superficie terrestre, y debido a sus características particulares como la baja disponibilidad de agua, son consideradas de gran importancia biológica, ecológica y socioeconómica (Ezcurra 2006, Huxman et al. 2004, Noy-Meir 1973, Prăvălie et al. 2019). Además de la aridez, otras condicionantes como alta irradiancia, temperaturas extremas y baja disponibilidad de nutrientes imponen otras limitantes a la funcionalidad de los organismos (Austin 2011, Gibson \& Rundel 2012). Esto conlleva a la necesidad de conocer las diferencias en las respuestas ecofisiológicas y morfoestructurales de las especies, en relación con las diferentes presiones selectivas ambientales, y su posible papel en la estructuración de las comunidades vegetales en estas regiones.

Las características morfológicas o fisiológicas que impactan positivamente la adecuación de las especies (atributos de respuesta) o afectan los procesos en los ecosistemas (atributos de efecto), son muy diversas y caracterizan tanto las estrategias adaptativas de las especies como la estructura de las comunidades (Bruelheide et al. 2018, Díaz \& Cabido 1997, Reich 2014, Violle et al. 2007). Algunos de los atributos funcionales se relacionan entre sí, dada la optimización de la energía y los recursos invertidos para la fotosíntesis y el crecimiento, tal como se reconocen en el espectro económico de la hoja (Chabot et al. 1982, Reich et al. 2003, Wright et al. 2004), que incluye procesos fisiológicos $\left(\mathrm{A}_{\max }=\right.$ Fotosíntesis máxima, $\mathrm{R}_{\text {mass }}=$ Respiración), concentración de nutrientes $\left(\mathrm{N}_{\text {area }}=\right.$ Nitrógeno por área, $\mathrm{N}_{\text {mass }}=$ Nitrógeno por masa, $\mathrm{P}_{\text {area }}=$ Fósforo por área, $\mathrm{P}_{\text {mass }}=$ Fósforo por masa) y componentes estructurales $(\mathrm{AFE}=$ Área foliar específica y $\mathrm{LL}=$ Longevidad de la hoja). Es notoria la falta del conocimiento existente sobre los atributos foliares de especies de regiones secas en las bases de datos globales (TRY, Kattge et al. 2011), a pesar de su importancia para entender las consecuencias del cambio climático en los modelos de los flujos de $\mathrm{C}$ y productividad globales.

La fotosíntesis es el atributo que mejor refleja la adecuación de las plantas al ambiente. Este proceso se ve negativamente afectado, principalmente en especies $\mathrm{C}_{3}$, cuando el agua es un factor limitante y perjudica sus componentes estomáticos y no estomáticos (Flexas \& Medrano 2002). Asimismo, la concentración de nitrógeno (N), componente de pigmentos como la clorofila y las enzimas de fijación del $\mathrm{CO}_{2}$, condiciona el proceso fotosintético (Evans \& Terashima 1987). Durante la fotosíntesis, el suministro de $\mathrm{CO}_{2}$ al interior de la hoja está optimizado por la apertura de los estomas que permiten al mismo tiempo la salida de vapor de agua del interior de la planta a la atmósfera, asegurando el uso eficiente del agua (Ball et al. 1987, Cowan \& Farquhar 1977, Flexas \& Medrano 2002, Gibson \& Rundel 2012, Granados-Sánchez et al. 1998). Particularmente, algunas especies en regiones secas disminuyen su apertura estomática para evitar la transpiración excesiva, aún a expensas de reducir su captación de $\mathrm{CO}_{2}$, e incrementar su capacidad de sobrevivencia durante los largos períodos de sequía (Flexas \& Medrano 2002, Gibson \& Rundel 2012).

La menor fijación de $\mathrm{CO}_{2}$ por limitación estomática y consecuente menor requerimiento de $\mathrm{N}$ en hojas, puede poner en conflicto la necesidad de soportar condiciones de alta irradiación que caracterizan a las regiones secas. Tales condiciones lumínicas requieren mayor concentración de $\mathrm{N}$ en las hojas para: a) aumentar la concentración de pigmentos ligados al atrapamiento de fotones utilizados en procesos fotosintéticos y no-fotosintéticos (Strasser et al. 2000, Taiz \& Zeiger 2006, Yamori 2016), b) el funcionamiento de vías metabólicas relacionadas con la fotoprotección (Ciompi et al. 1996, Živčák et al. 2014), c) requerimientos metabólicos asociados al mantenimiento de la integridad de los procesos fotoquímicos de la fotosíntesis (eficiencia cuántica del PSII, disipación y transporte de electrones, Baker (2008); d) la disipación energética (Björkman \& Demmig-Adams 1995, Demmig-Adams \& Adams 2006) y e) la reducción de $\mathrm{NO}_{3}$ (Andrews et al. 2013). Este tipo de disyuntiva parece ser común en especies de las regiones áridas de Norteamérica que muestran altas concentraciones de N en sus hojas (Skujins 1981, West \& Skujins 1978, Castellanos et al. 2018, Hinojo-Hinojo et al. 2018), así como en especies de la familia Fabaceae (= Leguminosae) y fijadoras de nitrógeno (Adams et al. 2016, Castellanos et al. 2018), lo que puede implicar la presencia de mayores tasas fotosintéticas (Hinojo-Hinojo et al. 2018), una mayor eficiencia en procesos de fotoprotección (Acuña-Acosta 2018), o inducir una mayor eficiencia en el uso de agua. En condiciones de estrés hídrico moderado, con aperturas estomáticas relativamente bajas, las altas concentraciones de $\mathrm{N}$ y tasas de fotosíntesis en las hojas parecen aumentar la eficiencia en el uso del agua en períodos cortos (Field et al. 1983), característica que parecen presentar las especies de leguminosas y algunas especies de regiones secas (Adams et al. 2016, Hinojo-Hinojo et al. 2018). Sin embargo, a pesar de los diversos estudios que han reportado altas concentraciones de $\mathrm{N}$ en hojas de especies desérticas y fijadoras de nitrógeno ( $\mathrm{NFj}$ ), sigue sin entenderse el papel que atributos relacionados con las altas concentraciones de $\mathrm{N}$ en las hojas puedan tener en 
la respuesta de las especies y en el funcionamiento de las comunidades vegetales de las regiones secas en el país (Rzedowski 2006, Laity 2008, Castellanos-Villegas et al. 2010, Franklin et al. 2016).

La diversidad de atributos funcionales de las especies puede converger a nivel de comunidad debido a las presiones selectivas bióticas o abióticas dominantes en la localidad (Bruelheide et al. 2018, Huxman et al. 2004). Aunque el agua se ha propuesto como el principal factor selectivo, la diferenciación de estrategias ecológicas con base en cocientes estequiométricos (Castellanos et al. 2018) y otros factores ambientales sugiere una mayor diversidad de respuestas de las especies en comunidades secas. Por ejemplo, además de la baja disponibilidad de humedad en el suelo, el nitrógeno y otros nutrientes pueden encontrarse de manera heterogénea y ser abundantes bajo el dosel de especies nodrizas, facilitando procesos microbiológicos y de mineralización (Celaya-Michel \& Castellanos-Villegas 2011, García-Moya \& McKell 1970, Medeiros \& Drezner 2012), sugiriendo diferencias en el balance de los elementos químicos clave como $\mathrm{C}, \mathrm{N}, \mathrm{P}$ y afectando no solo la ecofisiología de las plantas, sino también las interacciones ecológicas y la biogeoquímica de comunidades y ecosistemas (Sterner \& Elser 2002).

Conocer la eficiencia en el uso del agua o nutrientes de especies de zonas áridas es importante dados los futuros escenarios de incremento en la temperatura y la sequía con el cambio climático, que conllevan al incremento en las limitaciones de agua y nutrientes. Dichas limitantes afectarán procesos ecofisiológicos y atributos en las especies (Gago et al. 2014), modificarán la forma y características del ensamblado de las comunidades (Bruelheide et al. 2018) y tendrá efectos importantes en los procesos biogeoquímicos de los ecosistemas. En consecuencia, es crítico aumentar el conocimiento sobre los atributos funcionales de las plantas que permita mejorar las predicciones de los efectos ecofisiológicos ante los cambios ambientales y con ello mejorar la toma de decisiones relacionadas con la conservación de las especies y comunidades (Köhler et al. 2016, Ahrar et al. 2017).

Por lo tanto, el presente trabajo busca aportar más elementos para caracterizar los atributos foliares de especies $\mathrm{y}$ tipos funcionales de regiones secas, esclarecer su contribución a las estrategias de su uso eficiente del agua y/o nutrientes (nitrógeno y fósforo), y su papel en la conformación de las comunidades en que habitan. Para tal efecto se caracterizaron los atributos ecofisiológicos y estequiométricos relacionados con la fotosíntesis, la fotoprotección y la eficiencia en el uso del agua, $\mathrm{N}$ y $\mathrm{P}$ en especies y tipos funcionales del Desierto Sonorense, lo que permitió reconocer la diversidad de estrategias ecofisiológicas y estequiométricas que conforman dos de sus comunidades vegetales.

\section{Materiales y métodos}

Sitios de estudio. Se estudiaron 13 especies creciendo bajo condiciones naturales en dos comunidades vegetales de la región central de Sonora, México (Figura 1). Las dos comunidades se clasificaron según el índice de aridez de De Martonne utilizando datos de precipitación y temperatura de Worldclim (Fick \& Hijmans 2017). El primer sitio es el rancho El Churi (EC), se encuentra a $11.55 \mathrm{~km}$ del municipio de la Colorada, Sonora. Se ubica en las coordenadas $28^{\circ} 42^{\prime} 6.77^{\prime}$ 'N y $110^{\circ} 32$ ' 15.64 ” W. Presenta precipitación media anual de $400 \mathrm{~mm}$ y temperatura media anual de $22.59^{\circ} \mathrm{C}$. El grupo de suelo es un Regosol calcárico con textura media gravosa según datos de INEGI. La comunidad vegetal dominante dentro del rancho es Matorral subtropical (INEGI 2015). El otro sitio es el rancho San Judas (SJ), este se encuentra a $32.45 \mathrm{~km}$ de la ciudad de Hermosillo. Se ubica entre $29^{\circ} 17^{\prime} 50.10^{\prime \prime} \mathrm{N}$ y $111^{\circ} 10^{\prime}$ 8.60 " W. Presenta una precipitación media anual de 285 $\mathrm{mm}$, una temperatura media anual de $22.23{ }^{\circ} \mathrm{C}$. Dentro de este sitio dominan comunidades vegetales de matorral xerófilo, con mayor presencia de arbustos desérticos. El grupo de suelo según INEGI es un Xerosol háplico con una textura media gravosa.

Mediciones de atributos ecofisiológicos y estequiométricos en campo. El muestreo en campo se realizó durante el periodo de mayor actividad fotosintética, de agosto a septiembre de 2019 entre 8:00 y 11:00 am, cuando las hojas presentaron menor estrés durante el día. En ambos sitios se seleccionaron las especies dominantes (Tabla 1), un total de 8 especies en el sitio árido (San Judas) y 9 en el semiárido (El Churi) y 4 de esas especies fueron comunes en ambos sitios. Cada especie se clasificó en un tipo funcional basado en el tiempo de vida de la hoja y la capacidad de fijación de $\mathrm{N}$. La mayoría de las especies, en ambas comunidades, se clasificaron como caducifolias no fijadoras de nitrógeno, $50 \%$ en SJ y $55 \%$ en EC (Tabla 1 ). En $\mathrm{SJ}$, las especies fijadoras se encontraron en un $25 \%$ como perennifolias y $12.5 \%$ como caducifolias. En El Churi las especies fijadoras caducifolias y perennifolias estuvieron representadas con porcentajes iguales ( $22.5 \%$ cada una). En este sitio no se registraron especies perennifolias no fijadoras de nitrógeno. 


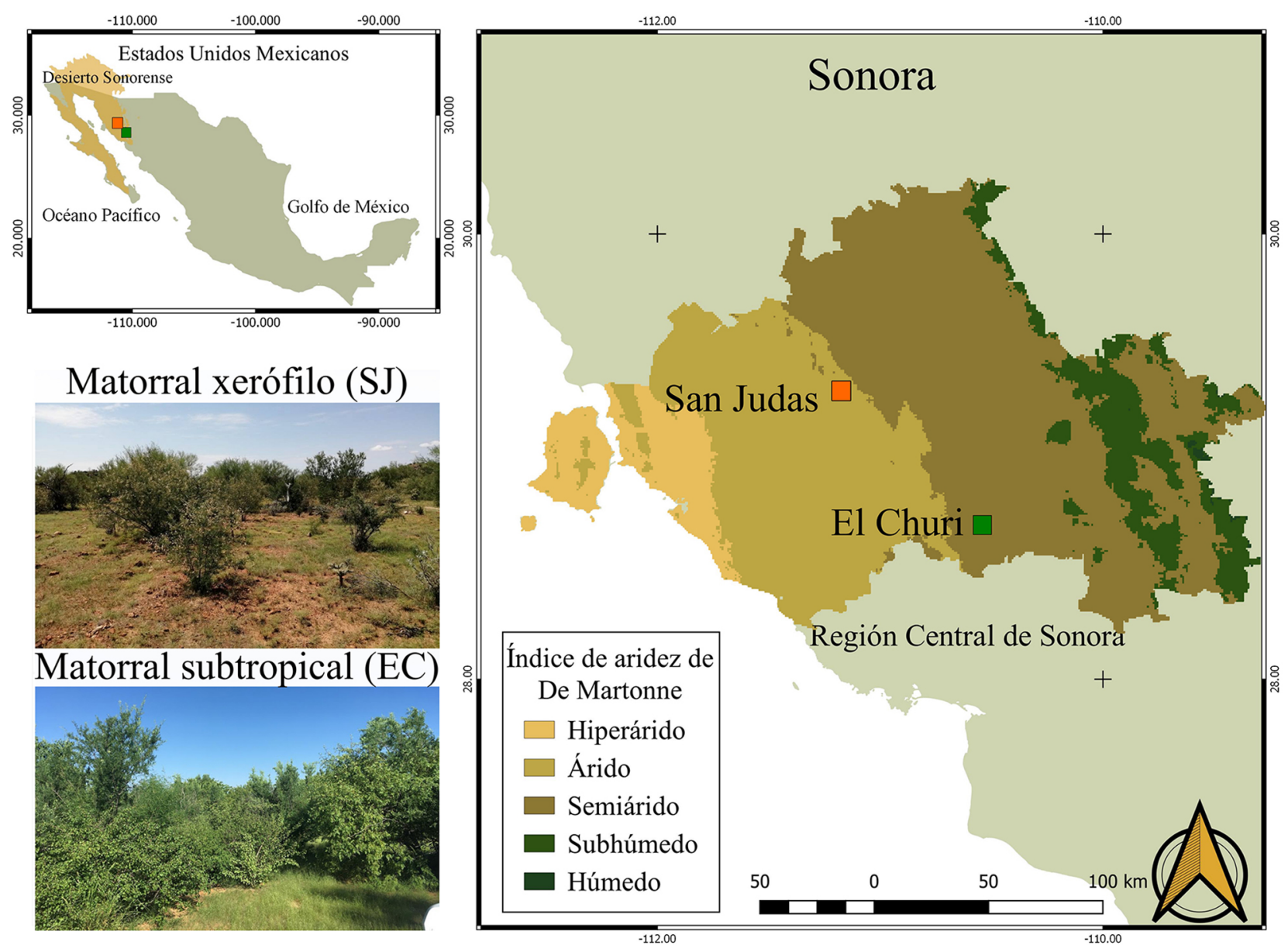

Figura 1. Sitios de estudio árido (SJ, anaranjado) y semiárido (EC, verde) en la región central de Sonora, México, mostrando la clasificación del índice de aridez de De Martonne.

De cada especie se marcaron de 3 a 5 individuos de manera aleatoria, a quienes se realizaron mediciones de fotosíntesis y fluorescencia de la clorofila por triplicado en hojas expuestas al sol y maduras; de los cuales, además, se colectaron muestras foliares en frío, manteniéndose así hasta posteriores determinaciones en laboratorio.

Atributos fotosintéticos. Se utilizó el sistema de fotosíntesis portátil LI6400XT (Licor, Lincoln, NE, USA) para determinar intercambio gaseoso en condiciones de campo. Se determinó la capacidad fotosintética neta $\left(\mathrm{A}_{\text {net }}=\right.$ cantidad de carbono fijado), conductancia estomática (gs), transpiración (Tr), curvas de respuesta a la luz y al $\mathrm{CO}_{2}$. El flujo de aire en la cámara fue de 300-500 $\mu \mathrm{mol} \mathrm{s}^{-1}$. Cada hoja medida se colectó para determinar el área foliar, corregir los valores de fotosíntesis y posterior determinación de nutrientes totales.

Las curvas de $\mathrm{CO}_{2}$ se modelaron mediante la función Fitaci del paquete de R ( $\mathrm{R}$ Core Team 2020) Planteco- phys utilizando el método "bilinear" (Duursma 2015), ajustando la temperatura a $25{ }^{\circ} \mathrm{C}$ para una comparación válida entre mediciones (Sharkey et al. 2007) y se modificó el set de parámetros de temperatura según Bernacchi et al. (2001), siguiendo lo recomendado por Hinojo-Hinojo et al. (2018). A partir de esto se determinaron los parámetros del modelo bioquímico de la fotosíntesis de Farquhar (Farquhar et al. 1980): $\mathrm{V}_{\text {cmax }}$ (velocidad de carboxilación máxima), $\mathrm{J}_{\max }$ (transporte de electrones máximo).

Las curvas de respuesta a la luz se elaboraron utilizando los puntos de mejor ajuste máximo de cada especie, por lo que no se presentan desviaciones estándar. Los parámetros se obtuvieron utilizando la ecuación de la recta a partir de la pendiente inicial $(0$ a $150 \mu \mathrm{mol}$ de fotones $\left.\mathrm{m}^{-2} \mathrm{~s}^{-1}\right)$ :

$$
\mathrm{y}=\mathrm{mx}+\mathrm{b}
$$


Donde la pendiente de la recta $(\mathrm{m})$ es el rendimiento cuántico de la fotosíntesis $\left(\phi, \mathrm{mol} \mathrm{CO}_{2}\right.$ mol fotones $\left.{ }^{-1}\right)$, el valor de la fotosíntesis cuando la irradiancia (x) es igual a cero es la tasa de respiración oscura ( $\left.\mathrm{Rd}, \mu \mathrm{mol} \mathrm{CO} \mathrm{m}^{-2} \mathrm{~s}^{-1}\right), \mathrm{y}$ el punto de compensación de la luz (PCL) es el valor de irradiancia cuando la diferencia entre fotosíntesis y respiración es cero.

Para graficar las curvas de respuesta a la luz se utilizó el modelo de ajuste a una parábola no rectangular, utilizando el código en R publicado por Heberling (2013). El modelo estima a través de la regresión de mínimos cuadrados no lineales los parámetros descritos en la siguiente ecuación:

$$
A_{n e t}=\frac{\phi P P F D+\sqrt{\left(\phi P P F D+A_{\max }\right)^{2}-4 \theta \phi P P F D+A_{\max }}}{2 \theta}-R o
$$

Donde $\mathrm{A}_{\text {net }} \mathrm{y}_{\text {max }}$ son las tasas de fotosíntesis neta basadas en área y de fotosíntesis gruesa $\left(\mu \mathrm{mol} \mathrm{CO} \mathrm{m}^{-2} \mathrm{~s}^{-1}\right)$ respectivamente, $\phi$ es el rendimiento cuántico aparente $\left(\mathrm{mol} \mathrm{CO}_{2}\right.$ mol fotones $\left.{ }^{-1}\right)$, Ro es la tasa de respiración en la oscuridad (fotosíntesis en ausencia de luz; $\mu \mathrm{mol} \mathrm{CO}_{2} \mathrm{~m}^{-2} \mathrm{~s}^{-1}$ ), y $\theta$ es el factor de curvatura de la curva (adimensional).

Atributos de la fluorescencia de la clorofila. A la par de las mediciones de intercambio gaseoso, se realizaron de- terminaciones de los parámetros de la fluorescencia de la clorofila, a 5 hojas por cada especie, con el equipo portátil Handy PEA (Hansatech Instruments Ltd, Kings Lynn, UK). Las hojas se adaptaron a la oscuridad por 30 minutos para oxidar los centros de reacción y dejar inactivos los fotosistemas II (Strasser et al. 2000).

Atributos relacionados con fotosíntesis. Se digitalizaron 3 hojas de cada individuo con un Scanner HP ${ }^{\circledR}$ Deskjet Ink Advantaje 2515 junto a una referencia de $4 \mathrm{~cm}^{2}$ y posteriormente se midió el área de la hoja con el software ImageJ v.1.48 (Schneider et al. 2012). Cada una de las hojas medidas se transfirieron etiquetadas a un horno $\mathrm{Fe}-$ lisa ${ }^{\circledR}$ modelo FE 243 a $65^{\circ} \mathrm{C}$ por 48 horas, para obtener el peso seco en una balanza analítica. El valor de área foliar específica (AFE) se obtiene mediante la división del área foliar de la hoja fresca entre el peso seco de la misma hoja y se expresa en $\mathrm{m}^{2} \mathrm{~kg}^{-1}$ (Perez-Harguindeguy et al. 2016).

Los pigmentos fotosintéticos, clorofilas y carotenos totales se determinaron a través de la extracción del pigmento, macerando la muestra vegetal con $15 \mathrm{~mL}$ de acetona al $80 \%$ en condiciones de oscuridad y temperatura fría; la medición se realizó utilizando un espectrofotómetro Genesys 20 (Thermo Scientific ${ }^{\circledR}$ Inc). Para clorofila $a$, se tomó lectura de la absorbancia a $645 \mathrm{~nm}$, para clorofila $b$ se ajustó

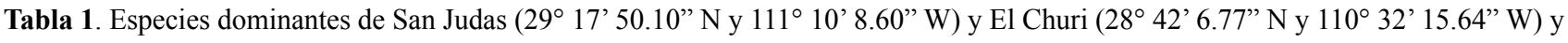
sus respectivos tipos funcionales. $\mathrm{TF}=$ Tipo funcional, $\mathrm{C}=$ Caducifolia, $\mathrm{P}=$ Perennifolia, $\mathrm{NF}=$ No fijadora de nitrógeno, $\mathrm{F}=\mathrm{Fijadora}$ de nitrógeno.

\begin{tabular}{|c|c|c|c|c|}
\hline Sitio & Especie & Familia & Nombre común & TF \\
\hline \multirow[t]{5}{*}{ El Churi } & Prosopis velutina Wooton. & Leguminosae & Mezquite & $\mathrm{PF}$ \\
\hline & Eysenhardtia orthocarpa (A. Gray) S. Watson & Leguminosae & Palo dulce & $\mathrm{CF}$ \\
\hline & Fouquieria macdougalii Nash & Fouquieriaceae & Ocotillo macho & $\mathrm{CNF}$ \\
\hline & Jatropha cordata (Ortega) Müll. Arg. & Euphorbiaceae & Papelillo & $\mathrm{CNF}$ \\
\hline & Ipomoea arborescens (Humb. \& Bonpl. ex Willd.) G. Don & Convolvulaceae & Palo santo & $\mathrm{CNF}$ \\
\hline \multirow[t]{4}{*}{ En común } & Encelia farinosa Torr. \& A. Gray & Asteraceae & Rama blanca & $\mathrm{CNF}$ \\
\hline & Jatropha cardiophylla Müll. Arg. & Euphorbiaceae & Sangrengado & $\mathrm{CNF}$ \\
\hline & Mimosa laxiflora Benth. & Leguminosae & Uña de gato & $\mathrm{CF}$ \\
\hline & Olneya tesota A. Gray & Leguminosae & Palo fierro & $\mathrm{PF}$ \\
\hline \multirow[t]{4}{*}{ San Judas } & Larrea tridentata $\mathrm{L}$. & Zygophyllaceae & Gobernadora & P NF \\
\hline & Cercidium microphyllum (Torr.) R. \& I. M. Johnst. & Leguminosae & Palo verde & $\mathrm{CNF}$ \\
\hline & Fouquieria diguetii (Tiegh.) I.M. Johnst. & Fouquieriaceae & Palo de Adán & $\mathrm{CNF}$ \\
\hline & Calliandra eriophylla Benth. & Leguminosae & Cósahui del norte & $\mathrm{CF}$ \\
\hline
\end{tabular}


a $663 \mathrm{~nm}$ y para carotenos totales a 480 y $750 \mathrm{~nm}$. Las fórmulas para las determinaciones de clorofilas y carotenos se basaron en Arnon (1949) y Guerra-Cantú et al. (2016).

Carbono, nitrógeno y fósforo total. Las hojas colectadas se secaron en un horno (Felisa ${ }^{\circledR}$ modelo FE 243 ) a $65{ }^{\circ} \mathrm{C}$ por 48 horas hasta peso constante y se homogeneizaron triturándolas en un molino Wiley con malla de 20 mesh. Se pesaron $3 \mathrm{mg}$ de muestra para determinar el contenido de carbono y nitrógeno total en un Analizador elemental PE$2400^{\circledR}$ SERIES II (Perkin-Elmer CHNS/O 2400, Waltham, USA) mediante combustión total a $950^{\circ} \mathrm{C}$ en presencia de oxígeno puro. El fósforo total se obtuvo por medio del proceso de digestión ácida con sulfato de cobre como catalizador y reacción colorimétrica con molibdato de amonio (Murphy \& Riley 1962), utilizando un analizador rápido de fluidos (SEAL, Norderstedt, Germany). Las concentraciones de $\mathrm{C}, \mathrm{N}$ y $\mathrm{P}$ se expresan por unidad de masa y con base en área mediante la división de la concentración de nutriente por el área foliar específica del individuo. Se incluye la lista de los atributos medidos con sus abreviaturas y unidades para mayor clarificación (Tabla 2).

Análisis estadístico. Todos los análisis se llevaron a cabo con un $95 \%$ de confianza en el software RStudio (RStudio Team 2020). Se realizaron pruebas de normalidad de Shapiro Wilk para definir si los datos se ajustaban a una distribución normal o no y, pruebas de homocedasticidad de Levene para definir igualdad de varianzas entre los grupos a analizar.

A los datos paramétricos se realizaron pruebas de $t$ para diferencias de medias entre las dos comunidades y ANOVA's para diferenciar tipos funcionales usando la función "aov" del paquete "stats", además de pruebas post hoc HSD de Tukey con la función HSD.test del paquete "agricolae". Para datos no paramétricos se utilizaron pruebas de Wilcoxon con la función "wilcox_test" del paquete "coin" para diferenciar medianas de las dos comunidades y Kruskal Wallis para diferenciar atributos entre los tipos funcionales. Se realizaron análisis de componentes principales con la función " $p c a$ " del paquete "factoextra" (Kassambara \& Mundt 2020) para determinar los atributos más importantes por comunidad y tipo funcional. Los datos no normales se transformaron a logaritmo base 10 para ajustarlos a una distribución normal y realizar análisis de correlación y regresión lineal. Finalmente, se evaluaron las correlaciones múltiples entre los atributos ecofisiológicos y estequiométricos, así como relaciones bivariadas entre dichos atributos por medio de un análisis de regresión lineal.

\section{Resultados}

Atributos ecofisiológicos y estequiométricos en las especies. Las tasas fotosintéticas $\left(\mathrm{A}_{\text {area }} \mathrm{y}_{\text {mass }}\right)$ entre las especies presentaron alta variabilidad de hasta 4 veces en sus tasas máximas. F. macdougalii tuvo la tasa media más baja mientras que E. farinosa y J. cardiophylla registraron los valores promedio más altos (Tabla 3 ). Las curvas de respuesta a la luz mostraron variación entre las especies de los sitios, con el punto de compensación de la luz (PLC) fluctuando de $22.8 \mu \mathrm{mol}$ fotones $\mathrm{m}^{2} \mathrm{~s}^{-1}$ en $P$. velutina a $130.0 \mu \mathrm{mol}$ fotones $\mathrm{m}^{2} \mathrm{~s}^{-1}$ en L. tridentata; mientras que los valores del punto de saturación de la luz (PSL) fluctuaron entre 255 y $1,869 \mu \mathrm{mol}$ fotones $\mathrm{m}^{2} \mathrm{~s}^{-1}$ (Figura 2). De la misma manera, los atributos de las curvas de respuesta al $\mathrm{CO}_{2}$ presentaron alta variabilidad entre las especies. $\mathrm{V}_{\text {cmax } 25}$ varió desde 44.6 a $177.6 \mu \mathrm{mol} \mathrm{m}^{-2} \mathrm{~s}^{-1}$ con los promedios más altos en $J$. cardiophylla y E. farinosa del sitio árido en San Judas (SJ), mientras que $\mathrm{J}_{\max 25}$ mostró tan solo en el sitio más árido (SJ) una diferencia de hasta 8 veces $\left(53.5\right.$ a $\left.477.8 \mu \mathrm{mol} \mathrm{m}^{-2} \mathrm{~s}^{-1}\right)$ en su rango de valores (Tabla 3).

$\mathrm{La}$ eficiencia en el uso del agua instantánea $\left(\mathrm{UEA}_{\text {inst }}=\right.$ $\mathrm{A}_{\text {area }} / \mathrm{Tr}$ ) presentó también un alto rango de variabilidad, de 9 veces entre las especies del sitio semiárido $(\mathrm{EC}=2.4$ $11.6 \mu \mathrm{mol} \mathrm{CO}_{2} \mathrm{mmol}^{-1} \mathrm{H}_{2} \mathrm{O}$ ) en donde O. tesota y M. laxiflora tuvieron los valores más altos, pero solo tres veces (2.5 - $6.2 \mu \mathrm{mol} \mathrm{CO}_{2} \mathrm{mmol}^{-1} \mathrm{H}_{2} \mathrm{O}$ ) en SJ (Tabla 3) . La eficiencia intrínseca en el uso del agua $\left(\mathrm{UEA}_{\text {intrins }}=\mathrm{A}_{\text {area }} / \mathrm{gs}\right)$ fluctuó de 74.5 a $199.4 \mu \mathrm{mol} \mathrm{CO}_{2} \mathrm{~mol}^{-1} \mathrm{H}_{2} \mathrm{O}$, con los promedios más altos en I. arborescens y J. cordata de EC (Tabla 3).

La eficiencia cuántica del PSII (Fv/Fm) y algunos de los componentes involucrados en la respuesta fotoquímica (ABS/CS, TRo/CS, y DIo/CS) tuvieron poca variación entre las especies. En cambio, los parámetros que indican la integridad de la respuesta, $\mathrm{PI}_{\text {abs y }} \mathrm{PI}_{\text {tot }}$, variaron casi 30 veces (Tabla 4), lo que es interesante pues la proporción de clorofila $a / b(\mathrm{Chl} a / b)$ varió menos del doble en su valor (2.36 - $\left.3.82 \mathrm{mg} \mathrm{L}^{-1}\right)$ entre las especies (Tabla 4). El promedio más bajo $(O$. tesota) y el más alto (E. orthocarpa) de carotenos totales, se presentó en especies fijadoras de nitrógeno.

En términos de nutrientes y cocientes estequiométri$\cos , \mathrm{N}_{\text {mass }}\left(11.7-39.3 \mathrm{mg} \mathrm{g}^{-1}\right)$ y $\mathrm{P}_{\text {mass }}\left(0.9-2.5 \mathrm{mg} \mathrm{g}^{-1}\right)$ fueron poco variables, al igual que los cocientes estequiométricos C:N (11.0 - 27.1) y N:P (8.9 - 26.9), sin embargo C:P (142.0 - 500.7) mostró amplios rangos de valores de 5 veces (Tabla 5). Aún así, la eficiencia en el uso del nitrógeno $\left(\mathrm{EUN}=\mathrm{A}_{\text {mass }} / \mathrm{N}_{\text {mass }}\right)$ varió entre 5.1 y 21.5, siendo 
Acuña-Acosta et al. / Botanical Sciences 99(2): 257-278. 2021

Tabla 2. Descripción de la nomenclatura de atributos funcionales y sus unidades utilizados en este estudio.

\begin{tabular}{|c|c|c|c|c|}
\hline & & Descripción & Atributo & Unidades \\
\hline \multirow{12}{*}{\multicolumn{2}{|c|}{ 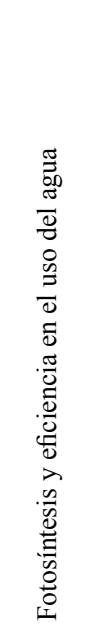 }} & Tasa de fotosíntesis con base en área & $\mathrm{A}_{\text {area }}$ & $\mu \mathrm{mol} \mathrm{CO}{ }_{2} \mathrm{~m}^{-2} \mathrm{~s}^{-1}$ \\
\hline & & Tasa de fotosíntesis con base en masa & $A_{\text {mass }}$ & $\mu \mathrm{mol} \mathrm{CO} \mathrm{g}^{-1} \mathrm{~s}^{-1}$ \\
\hline & & Rendimiento cuántico de la fotosíntesis & $\varphi$ & $\mu \mathrm{mol} \mathrm{CO}{ }_{2} \mu \mathrm{mol}^{-1}$ fotón \\
\hline & & Punto de compensación de la luz & PLC & $\mu$ mol fotón $\mathrm{m}^{-2} \mathrm{~s}^{-1}$ \\
\hline & & Punto de saturación de la luz & PSL & $\mu \mathrm{mol}$ fotón $\mathrm{m}^{-2} \mathrm{~s}^{-1}$ \\
\hline & & Tasa de respiración oscura & Ro & $\mu \mathrm{mol} \mathrm{CO}{ }_{2} \mathrm{~m}^{-2} \mathrm{~s}^{-1}$ \\
\hline & & Velocidad de carboxilación máxima & $\mathrm{V}_{\mathrm{cmax} 25}$ & $\mu \mathrm{mol} \mathrm{CO} \mathrm{m}^{-2} \mathrm{~s}^{-1}$ \\
\hline & & Transporte de electrones & $\mathrm{J}_{\max 25}$ & $\mu \mathrm{mol} \mathrm{CO}{ }_{2} \mathrm{~m}^{-2} \mathrm{~s}^{-1}$ \\
\hline & & Transpiración & $\operatorname{Tr}$ & $\mathrm{mmol} \mathrm{H}_{2} \mathrm{O} \mathrm{m}^{-2} \mathrm{~s}^{-1}$ \\
\hline & & Conductancia estomática & gs & $\mathrm{mol} \mathrm{H}_{2} \mathrm{O} \mathrm{m}^{-2} \mathrm{~s}^{-1}$ \\
\hline & & Eficiencia en el uso del agua instantánea & UEA $_{\text {inst }}$ & $\mu \mathrm{mol} \mathrm{CO}{ }_{2} \mu \mathrm{mol}^{-1} \mathrm{H}_{2} \mathrm{O}$ \\
\hline & & Eficiencia en el uso del agua intrínseca & UEA $_{\text {intrins }}$ & $\mu \mathrm{mol} \mathrm{CO} \mathrm{mol}^{-1} \mathrm{H}_{2} \mathrm{O}$ \\
\hline \multirow{17}{*}{\multicolumn{2}{|c|}{ 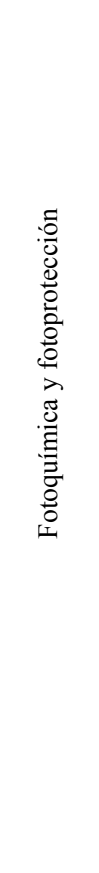 }} & Carotenos totales & Car tot & $\operatorname{mg~L}{ }^{-1}$ \\
\hline & & Clorofila total & Chl tot & $\mathrm{mg} \mathrm{L}^{-1}$ \\
\hline & & Concentración de clorofila $a$ & Chl $a$ & $\mathrm{mg} \mathrm{g}^{-1}$ \\
\hline & & Concentración de clorofila $b$ & $\mathrm{Chl} b$ & $\mathrm{mg} \mathrm{g}^{-1}$ \\
\hline & & Proporción de clorofila $a$ y $b$ & Chl $a / b$ & - \\
\hline & & Área foliar específica & AFE & $\mathrm{kg} \mathrm{m}^{-2}$ \\
\hline & & Eficiencia cuántica del PSII & $\mathrm{Fv} / \mathrm{Fm}$ & - \\
\hline & & Índice de funcionamiento por absorción & $\mathrm{PI}_{\mathrm{abs}}$ & - \\
\hline & & Índice de funcionamiento total & $\mathrm{PI}_{\text {tot }}$ & - \\
\hline & & Centros de reacción por absorción & $\mathrm{RC} / \mathrm{ABS}$ & - \\
\hline & & Centros de reacción por área & $\mathrm{RC} / \mathrm{CS}$ & - \\
\hline & & Flujo de fotones absorbidos & $\mathrm{ABS} / \mathrm{CS}$ & - \\
\hline & & Flujo de fotones atrapados & TRo/CS & - \\
\hline & & Flujo de fotones disipados & $\mathrm{DIo} / \mathrm{CS}$ & - \\
\hline & & Flujo de transporte de electrones & $\mathrm{ETo} / \mathrm{CS}$ & - \\
\hline & & Carbono foliar basado en masa & $\mathrm{C}_{\text {mass }}$ & $\mathrm{mg} \mathrm{g}^{-1}$ \\
\hline & & Nitrógeno foliar basado en masa & $\mathrm{N}_{\text {mass }}$ & $\mathrm{mg} \mathrm{g}^{-1}$ \\
\hline \multirow{8}{*}{\multicolumn{2}{|c|}{ 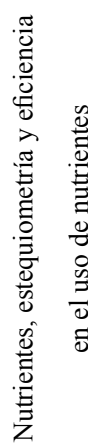 }} & Fósforo foliar basado en masa & $P_{\text {mass }}$ & $\mathrm{mg} \mathrm{g}^{-1}$ \\
\hline & & Carbono foliar basado en área & $\mathrm{N}_{\text {area }}$ & $\mathrm{g} \mathrm{N} \mathrm{m}^{-2}$ \\
\hline & & Fósforo foliar basado en área & $P_{\text {area }}$ & $\mathrm{g} \mathrm{P} \mathrm{m}^{-2}$ \\
\hline & & Proporción carbono/nitrógeno foliar & $\mathrm{C}: \mathrm{N}$ & - \\
\hline & & Proporción carbono/fósforo & $\mathrm{C}: \mathrm{P}$ & - \\
\hline & & Proporción nitrógeno/fósforo & $\mathrm{N}: \mathrm{P}$ & - \\
\hline & & Eficiencia en el uso del fósforo & EUP & $\mu \mathrm{mol} \mathrm{CO} \mathrm{mg}^{-1} \mathrm{P} \mathrm{s}^{-1}$ \\
\hline & & Eficiencia en el uso del nitrógeno & EUN & $\mu \mathrm{mol} \mathrm{CO} \mathrm{mg}^{-1} \mathrm{~N} \mathrm{~s}^{-1}$ \\
\hline
\end{tabular}


$F$. diguetii y E. farinosa de SJ las que presentaron los promedios más altos (Tabla 5). En el caso de la eficiencia en el uso del fósforo (EUP) la media menor se registró en $J$. cordata (0.41) en EC y la mayor en $F$. diguetii (2.54) en el sitio árido (SJ).

Diferencias de los atributos entre las dos comunidades. Se establecieron las diferencias a nivel comunidad para analizar el efecto de la aridez sobre el conjunto de los atributos de las especies en cada comunidad. Los atributos mostraron gran variabilidad como se mencionó antes y solo 9 de 32 atributos analizados resultaron significativamente diferentes entre las dos comunidades, ninguno relacionado con atributos de la fotosíntesis $\left(\mathrm{A}_{\text {area }}, \mathrm{A}_{\text {mass }}, \mathrm{gs}\right.$, $\operatorname{Tr}, \mathrm{V}_{\text {cmax } 25}$ ), aun cuando tendieron a presentar promedios más altos en el sitio con más aridez (SJ, Tabla 6). Las principales diferencias se relacionaron con parámetros de los procesos fotoquímicos de absorción y atrapamiento de fotones (ABS/CS y TRo/CS) y las concentraciones de nutrientes ( $\mathrm{N}$ y P) que fueron mayores en las hojas del sitio semiárido (EC). Otros parámetros relacionados con los centros de reacción como RC/ABS $(t=-2.23, \mathrm{df}=74.3$, $P=0.028)$, chl $a / b(t=2.62, \mathrm{df}=46, P=0.01)$ así como los cocientes estequiométricos $\mathrm{C}: \mathrm{N}(Z=-2.17, P=0.029)$ y C:P $(t=2.97, \mathrm{df}=31, P=0.005)$ fueron mayores en el sitio árido (SJ).

Relaciones bivariadas de atributos entre los sitios. Los análisis de relaciones bivariadas se obtuvieron a partir de las medias de las especies en cada comunidad y se obtuvo también el coeficiente de determinación general para todo el conjunto de todas las especies de ambas comunidades. Por ejemplo, aun cuando existieron diferencias en los coeficientes de determinación de $\mathrm{A}_{\text {area }} v s \operatorname{Tr} \mathrm{y}_{\text {area }} v s$ gs entre SJ $\left(R^{2}=0.71, P=0.007\right.$ y $\left.R^{2}=0.62, P=0.003\right)$ y EC $\left(R^{2}=0.43\right.$ y $\left.R^{2}=0.27\right)$, las pendientes no fueron diferentes, indicando que en ambos sitios mayores tasas de fotosíntesis generan incrementos en la transpiración
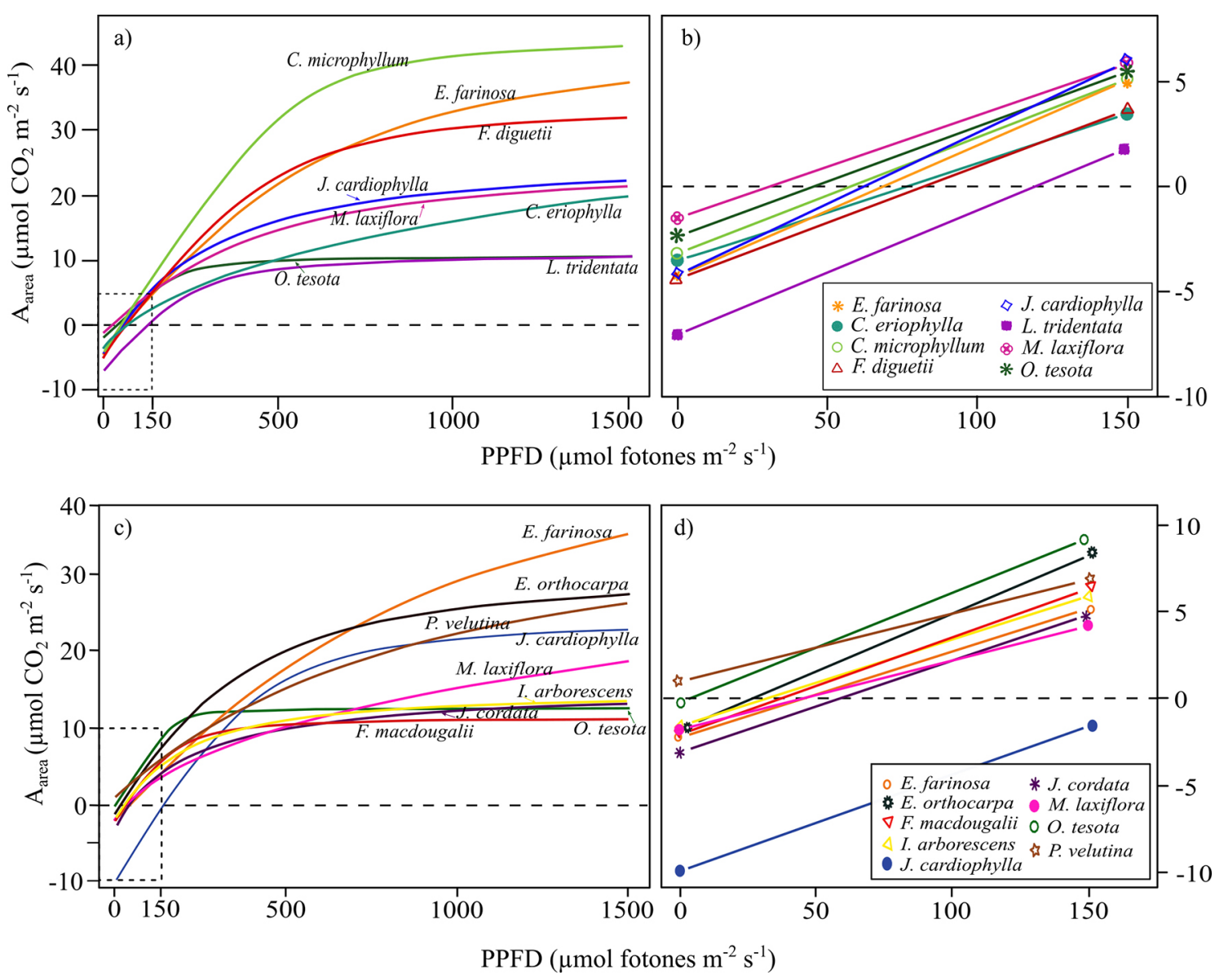

Figura 2. Curvas de respuesta fotosintética a la luz y pendientes iniciales de las especies en la comunidad árida (SJ, a y b) y semiárida (EC, c y d). Las curvas se modelaron con los datos del mejor ajuste de tres individuos de cada especie. 
$\left(\mathrm{UEA}_{\text {inst }}\right)$ y la conductancia estomática $\left(\mathrm{UEA}_{\text {intrins }}\right)$. Las mayores correlaciones vincularon los procesos de fotosíntesis con fotoprotección en el sitio árido. Por ejemplo, la relación $\mathrm{A}_{\text {area }}$ vs $\mathrm{DIo} / \mathrm{CS}$ presentó correlación inversa y significativa en ambos sitios, pero el coeficiente de determinación fue más alto en $\mathrm{SJ}\left(R^{2}=0.66, P=0.013\right.$, Figura 3). Igualmente, en $\mathrm{SJ}$ se registraron correlaciones altas y significativas entre $\mathrm{Fv} / \mathrm{Fm} v s$ gs $\left(R^{2}=0.80, P=0.002\right)$ y $\mathrm{A}_{\text {area }} v s$ Chl $a\left(R^{2}=0.71, P=0.007\right)$, y solo en EC la relación $\mathrm{Fv} / \mathrm{Fm} v s \mathrm{~A}_{\text {area }}$ presentó un mayor ajuste $\left(R^{2}=0.49\right.$, $P=0.03)$. En cambio, las relaciones asociadas al espectro económico de la hoja, fueron escasas, solo positiva entre AFE $v s \mathrm{~N}_{\text {mass }}\left(R^{2}=0.59 P=0.02\right)$ en $\mathrm{SJ}$ y negativa entre AFE $v s \mathrm{~N}_{\text {area }}\left(R^{2}=0.78, P=0.002\right)$ en EC (Figura 3), demostrando así diferencias importantes y únicas en las respuestas bivariadas de atributos de las especies de los dos sitios.
Tendencias multivariadas de atributos ecofisiológicos y estequiométricos entre las dos comunidades. El análisis de componentes principales (ACP) permitió diferenciar los atributos que más contribuyeron a explicar la varianza entre las dos comunidades vegetales, árida y semiárida. El total de la varianza explicada por los primeros dos componentes fue de $73.3 \%$ (Figura 4). El primer componente $(x)$ se asoció positivamente con la disipación de fotones y negativamente con las tasas de fotosíntesis, la EUN y el rendimiento fotoquímico. El segundo componente $(y)$ se asoció positivamente con los cocientes estequiométricos y la eficiencia en el uso de los nutrientes, y de manera negativa con UEA $\mathrm{Anst}_{\text {i }} \mathrm{y} \mathrm{N}_{\text {mass }}$. La mayor carga hacia el eje negativo del primer componente se relaciona con el sitio árido (SJ), asociado a las tasas de fotosíntesis, rendimiento fotoquímico y eficiencia en el uso de los nutrientes. En cambio, la comunidad semiárida (EC) presentó carga hacia el eje negativo del segundo componente
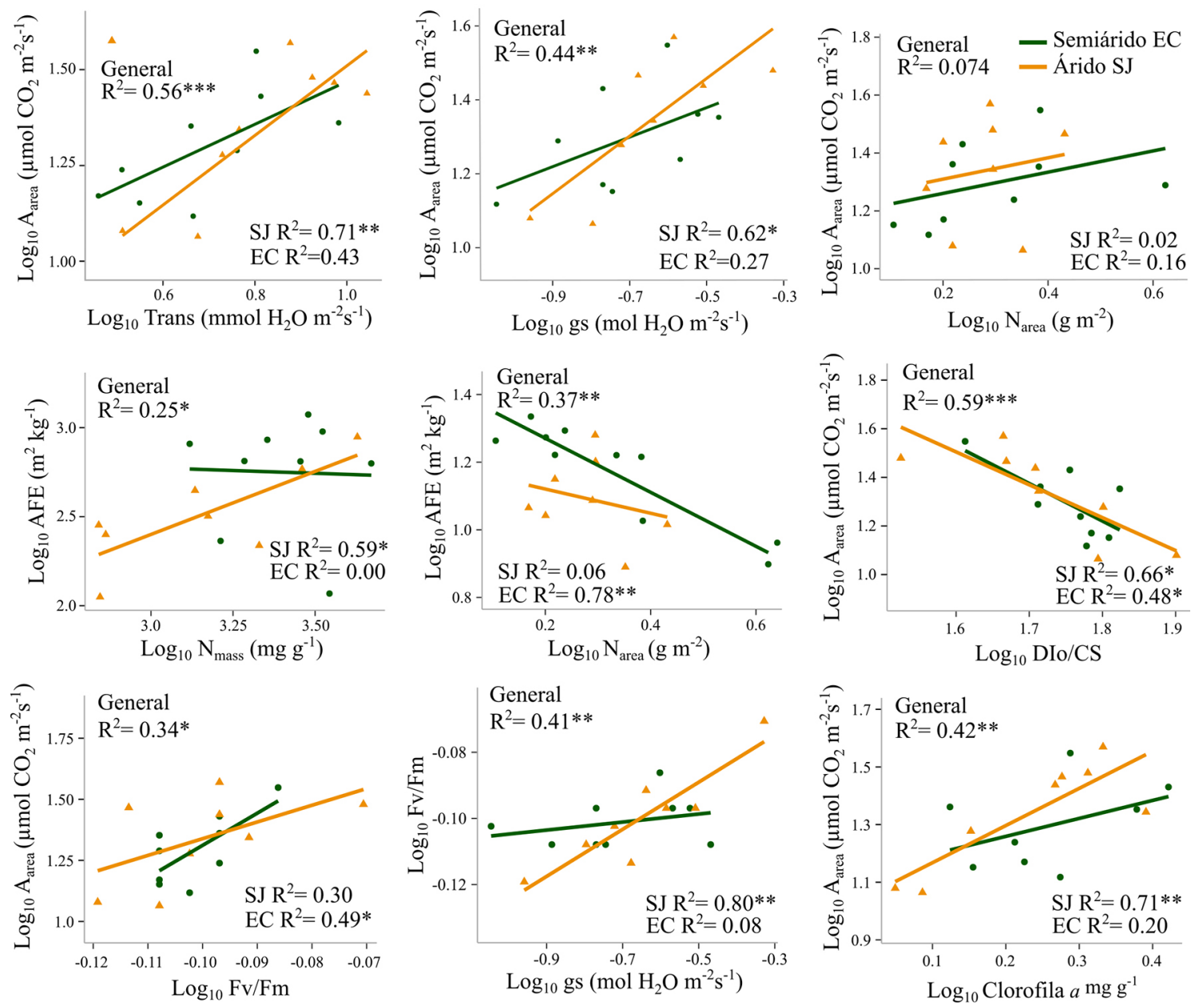

Figura 3. Relaciones bivariadas entre atributos funcionales transformados a logaritmo base 10 en las especies de San Judas (SJ) y El Churi (EC) y para todas las especies de las dos comunidades en general. Significancia estadística $P=0.05(*), P=<0.01(* *), P=<0.001(* * *)$. 
que se asoció principalmente a $\mathrm{N}_{\text {mass }}, \mathrm{UEA}_{\text {inst }} \mathrm{y} \mathrm{PI}_{\text {tot }}, \mathrm{y}$ tuvo carga positiva con el primer componente asociado a DIo/CS.

\section{Discusión}

Este estudio amplía en gran medida el conocimiento existente sobre los atributos ecofisiológicos y estequio- métricos de especies dominantes y tipos funcionales del Desierto Sonorense, comparándolas entre dos de sus comunidades vegetales, una árida y otra semiárida, ubicadas en la región central de Sonora. Además, busca resolver la siguiente hipótesis: los atributos estudiados, confieren una mayor eficiencia en el uso de agua a las especies y tipos funcionales de la comunidad más árida,

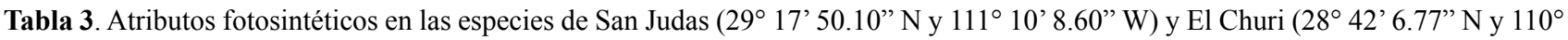
32 ' 15.64” W). $\mathrm{A}_{\text {area }}=$ Fotosíntesis con base en área $\left(\mu \mathrm{mol} \mathrm{CO} \mathrm{m}^{-2} \mathrm{~s}^{-1}\right), \mathrm{A}_{\text {mass }}=$ Fotosíntesis con base en masa $\left(\mu \mathrm{mol} \mathrm{CO} \mathrm{g}^{1} \mathrm{~s}^{1}\right), \mathrm{AFE}=$ área foliar específica $\left(\mathrm{m}^{2} \mathrm{~kg}^{-1}\right), \varphi=$ rendimiento cuántico de la fotosíntesis $\left(\mu \mathrm{mol} \mathrm{CO}{ }_{2} \mu \mathrm{mol}^{-1}\right.$ fotones), Ro $=$ respiración en oscuridad $(\mu \mathrm{mol}$ $\left.\mathrm{CO}_{2} \mathrm{~m}^{-2} \mathrm{~s}^{-1}\right), \mathrm{V}_{\text {cmax } 25}=$ velocidad de carboxilación máxima a $25^{\circ} \mathrm{C}\left(\mu \mathrm{mol} \mathrm{m} \mathrm{m}^{-2} \mathrm{~s}^{-1}\right), \mathrm{J}_{\max 25}=$ transporte de electrones a $25^{\circ} \mathrm{C}\left(\mu \mathrm{mol} \mathrm{m}{ }^{-2} \mathrm{~s}^{-1}\right)$, $\mathrm{UEA}_{\text {inst }}=$ Eficiencia en el uso del agua instantánea $\left(\mu \mathrm{mol} \mathrm{CO} \mathrm{mmol}^{-1} \mathrm{H}_{2} \mathrm{O}\right)$ y $\mathrm{UEA}_{\text {intrins }}=$ Eficiencia en el uso del agua instrínseca $(\mathrm{mol}$ $\left.\mathrm{CO}_{2} \mathrm{~mol}^{1} \mathrm{H}_{2} \mathrm{O}\right) . \mathrm{n}=$ de 3 a 5 datos por especie. $\mathrm{NA}=$ No aplica.

\begin{tabular}{|c|c|c|c|c|c|c|c|c|c|c|}
\hline Sitio & Especie & $\mathbf{A}_{\text {area }}$ & $\mathbf{A}_{\text {mass }}$ & AFE & $\varphi$ & Ro & $V_{\text {cmax25 }}$ & $\mathbf{J}_{\max 25}$ & $\mathbf{U E A}_{\text {inst }}$ & $\mathbf{U E A}_{\text {intrins }}$ \\
\hline \multirow{13}{*}{$\begin{array}{l}\text { El } \\
\text { Churi }\end{array}$} & Encelia farinosa & $35.3 \pm 3.0$ & $0.31 \pm \mathrm{NA}$ & $10.6 \pm 1.8$ & 0.047 & 2.14 & $80.4 \pm 13.7$ & $112.2 \pm \mathrm{NA}$ & $5.6 \pm \mathrm{NA}$ & $140.2 \pm \mathrm{NA}$ \\
\hline & Eysenhardtia & $26.9 \pm 0.6$ & $0.13 \pm \mathrm{NA}$ & $19.6 \pm 1.2$ & 0.065 & 1.69 & $65.7 \pm 4$ & $104.6 \pm 9.04$ & $4.2 \pm \mathrm{NA}$ & $155.0 \pm \mathrm{NA}$ \\
\hline & orthocarpa & & & & & & & & & \\
\hline & Fouquieria & $14.2 \pm 4.8$ & $0.08 \pm 0.02$ & $18.3 \pm 1.1$ & 0.054 & 2 & $90.8 \pm 10.9$ & $133.7 \pm 21.6$ & $6.3 \pm 4.7$ & $184.8 \pm 183.1$ \\
\hline & macdougalii & & & & & & & & & \\
\hline & Ipomoea & $13.1 \pm 1.2$ & $0.06 \pm 0.01$ & $21.6 \pm 1.9$ & 0.048 & 1.52 & $83.8 \pm 6.5$ & $131.1 \pm 23.2$ & $3.4 \pm 1.9$ & $199.4 \pm 152.8$ \\
\hline & arborescens & & & & & & & & & \\
\hline & Jatropha & $22.9 \pm 4.1$ & $0.14 \pm \mathrm{NA}$ & $16.6 \pm 2.6$ & 0.054 & 9.88 & $89.7 \pm 3.1$ & $144.5 \pm \mathrm{NA}$ & $2.4 \pm \mathrm{NA}$ & $76.4 \pm \mathrm{NA}$ \\
\hline & cardiophylla & & & & & & & & & \\
\hline & Jatropha cordata & $14.8 \pm 2.4$ & $0.08 \pm 0.01$ & $18.8 \pm 2.4$ & 0.051 & 2.99 & $81.4 \pm 4.9$ & $130.9 \pm \mathrm{NA}$ & $6.4 \pm 3.5$ & $191.1 \pm \mathrm{NA}$ \\
\hline & Mimosa laxiflora & $22.5 \pm 5.7$ & $0.14 \pm 0.03$ & $16.4 \pm 2.8$ & 0.047 & 1.7 & $94.4 \pm 21.7$ & $393.9 \pm \mathrm{NA}$ & $7.7 \pm 5.4$ & $74.5 \pm 29.6$ \\
\hline & Olneya tesota & $17.3 \pm 3.0$ & $0.11 \pm 0.02$ & $16.6 \pm 2.2$ & 0.063 & 0.21 & $112.7 \pm 26.4$ & $118.3 \pm \mathrm{NA}$ & $11.6 \pm 11.6$ & $139.2 \pm 138.8$ \\
\hline & Prosopis velutina & $19.5 \pm 9.1$ & $0.25 \pm 0.1$ & $7.9 \pm 0.5$ & 0.038 & -0.88 & $101.8 \pm 52.2$ & $126.9 \pm \mathrm{NA}$ & $3.6 \pm 2.3$ & $157.7 \pm 93.8$ \\
\hline \multirow{12}{*}{$\begin{array}{l}\text { San } \\
\text { Judas }\end{array}$} & Calliandra & $18.9 \pm 1.1$ & $0.16 \pm 0.01$ & $11.6 \pm 0.5$ & 0.0533 & 3.54 & $79.4 \pm 9.5$ & $100.9 \pm 78.1$ & $3.5 \pm 0.3$ & $108.3 \pm 41.5$ \\
\hline & eriophylla & & & & & & & & & \\
\hline & Cercidium & $29.2 \pm 20.4$ & $0.29 \pm 0.20$ & $10.4 \pm 0.9$ & 0.055 & 3.19 & $44.6 \pm 16.4$ & $53.5 \pm \mathrm{NA}$ & $3.0 \pm 1.6$ & $133.6 \pm 74.8$ \\
\hline & microphyllum & & & & & & & & & \\
\hline & Encelia farinosa & $37.1 \pm 1.4$ & $0.31 \pm \mathrm{NA}$ & $12.2 \pm 1.1$ & 0.062 & 4.28 & $177.6 \pm 15.4$ & $477.8 \pm \mathrm{NA}$ & $4.9 \pm \mathrm{NA}$ & $143.5 \pm \mathrm{NA}$ \\
\hline & Fouquieria & $27.4 \pm 7.3$ & $0.24 \pm 0.06$ & $11.0 \pm 0.4$ & 0.053 & 4.35 & $113.6 \pm 36.8$ & $139.3 \pm \mathrm{NA}$ & $2.5 \pm 0.8$ & $106.3 \pm 76.4$ \\
\hline & diguetii & & & & & & & & & \\
\hline & Jatropha & $30.2 \pm 10.8$ & $0.16 \pm 0.05$ & $19.1 \pm 1.1$ & 0.067 & 4.21 & $140 \pm 8.70$ & $140.7 \pm \mathrm{NA}$ & $4.3 \pm 1.9$ & $105.0 \pm 82.2$ \\
\hline & cardiophylla & & & & & & & & & \\
\hline & Larrea tridentata & $11.6 \pm 0.8$ & $0.15 \pm 0.01$ & $7.8 \pm 0.8$ & 0.055 & 7.19 & $63.3 \pm 9.0$ & $101.7 \pm 21.1$ & $2.8 \pm 1.1$ & $76.1 \pm 25.5$ \\
\hline & Mimosa laxiflora & $22.1 \pm 2.5$ & $0.14 \pm 0.01$ & $15.9 \pm 1.2$ & 0.049 & 1.47 & $109.7 \pm 15.7$ & $130.3 \pm 1.8$ & $6.2 \pm 4.0$ & $160.3 \pm 112.9$ \\
\hline & Olneya tesota & $12.0 \pm 0.8$ & $0.08 \pm 0.01$ & $14.12 \pm 1.43$ & 0.052 & 2.38 & $68.4 \pm 9.1$ & $76.4 \pm 16.1$ & $3.8 \pm 0.9$ & $154.6 \pm 119.5$ \\
\hline
\end{tabular}


$\mathrm{y}$ esos mismos atributos una mayor eficiencia en el uso de nutrientes en las especies y tipos funcionales de la comunidad semiárida. No obstante, este estudio revela que la hipótesis no fue válida en la escala de las comunidades, reflejando así posiblemente las diferencias en las interacciones ecológicas y la influencia de otras presiones selectivas locales diferentes a las limitantes de agua, en la determinación de las eficiencias en el uso de los recursos.
Efecto de la aridez en los atributos relacionados a la eficiencia en el uso del agua. Es ampliamente conocido que la UEA, tiene una fuerte relación con la aridez, tanto a nivel global como nivel comunidad (Ito \& Inatomi 2012, Nolan et al. 2018, Marozas et al. 2019), y es considerado un atributo económico crítico para las plantas en comunidades áridas (Ávila-Lovera et al. 2019). Debido a esto, se esperaba encontrar, en promedio, mayor UEA (UEA inst $_{\mathrm{y}} \mathrm{y}$ $U_{E A_{\text {intrins }}}$ ) en las especies del sitio con mayor aridez, pero

Tabla 4. Atributos de la fluorescencia de la clorofila y pigmentos fotosintéticos en las especies de San Judas $\left(29^{\circ} 17^{\prime} 50.10^{\prime \prime} \mathrm{N}\right.$ y $111^{\circ}$ $10^{\prime} 8.60^{\prime}$ W) y El Churi (2842’ 6.77” N y $110^{\circ} 32$ ' 15.64” W). Fv/Fm = eficiencia cuántica del fotosistema II, PI ${ }_{\text {abs }}=$ índice de funcionamiento por absorción, $\mathrm{PI}_{\text {tot }}=$ índice de funcionamiento total. Chl $a / b=$ Proporción clorofila $a / b$, Car $=$ carotenos totales $\left(\mathrm{mg} \mathrm{L}^{-1}\right) . \mathrm{Se}$ presentan la media \pm desviación estándar. $\mathrm{n}=$ de 3 a 5 datos por especie. NA $=$ No aplica.

\begin{tabular}{|c|c|c|c|c|c|c|c|c|c|}
\hline Sitio & Especie & Fv/Fm & $\mathbf{P I}_{\mathrm{abs}}$ & $P I_{\text {tot }}$ & $\mathrm{ABS} / \mathrm{CS}$ & TRo/CS & DIo/CS & Chl a/b & Car \\
\hline \multirow{9}{*}{$\begin{array}{l}\text { El } \\
\text { Churi }\end{array}$} & Encelia farinosa & $0.82 \pm 0.02$ & $10.9 \pm 4.7$ & $13.3 \pm 5.2$ & $219.4 \pm 23.1$ & $178.5 \pm 17.3$ & $40.9 \pm 6.5$ & $3.15 \pm 0.31$ & $1.75 \pm 0.45$ \\
\hline & $\begin{array}{l}\text { Eysenhardtia } \\
\text { orthocarpa }\end{array}$ & $0.80 \pm 0.02$ & $5.5 \pm 1.8$ & $7.4 \pm 2.2$ & $280.0 \pm 29.4$ & $223.1 \pm 20.6$ & $56.9 \pm 10.2$ & $2.36 \pm 0.33$ & $2.65 \pm 0.43$ \\
\hline & $\begin{array}{l}\text { Fouquieria } \\
\text { macdougalii }\end{array}$ & $0.78 \pm 0.06$ & $5.0 \pm 3.0$ & $6.8 \pm 3.8$ & $277.4 \pm 49.3$ & $213.0 \pm 21.2$ & $64.4 \pm 30.0$ & $3.03 \pm 0.46$ & $1.70 \pm 0.77$ \\
\hline & $\begin{array}{l}\text { Ipomoea } \\
\text { arborescens }\end{array}$ & $0.79 \pm 0.06$ & $4.9 \pm 2.2$ & $6.6 \pm 2.9$ & $282.4 \pm 32.7$ & $222.4 \pm 13.4$ & $60.0 \pm 23.6$ & $3.08 \pm 0.34$ & $2.02 \pm 0.47$ \\
\hline & $\begin{array}{l}\text { Jatropha } \\
\text { cardiophylla }\end{array}$ & $0.80 \pm 0.05$ & $7.0 \pm 3.8$ & $9.0 \pm 4.5$ & $258.0 \pm 32.0$ & $206.1 \pm 22.3$ & $51.9 \pm 17.4$ & $3.03 \pm \mathrm{NA}$ & $1.58 \pm \mathrm{NA}$ \\
\hline & Jatropha cordata & $0.78 \pm 0.07$ & $8.4 \pm 5.9$ & $10.3 \pm 6.9$ & $274.0 \pm 37.4$ & $213.1 \pm 23.1$ & $60.9 \pm 28.6$ & $3.48 \pm 0.45$ & $2.00 \pm 0.56$ \\
\hline & Mimosa laxiflora & $0.78 \pm 0.04$ & $7.0 \pm 3.9$ & $8.8 \pm 4.6$ & $291.7 \pm 78.7$ & $225.0 \pm 52.3$ & $66.6 \pm 27.9$ & $2.92 \pm 0.29$ & $2.50 \pm 0.16$ \\
\hline & Olneya tesota & $0.80 \pm 0.04$ & $6.1 \pm 3.1$ & $8.3 \pm 3.9$ & $286.1 \pm 36.8$ & $227.4 \pm 17.8$ & $58.9 \pm 21.7$ & $2.97 \pm 0.17$ & $1.41 \pm 0.32$ \\
\hline & Prosopis velutina & $0.78 \pm 0.04$ & $7.0 \pm 4.5$ & $8.9 \pm 5.2$ & $228.8 \pm 40.8$ & $177.2 \pm 23.5$ & $51.5 \pm 17.9$ & NA & NA \\
\hline \multirow[t]{8}{*}{$\begin{array}{l}\text { San } \\
\text { Judas }\end{array}$} & $\begin{array}{l}\text { Calliandra } \\
\text { eriophylla }\end{array}$ & $0.79 \pm 0.03$ & $5.6 \pm 1.9$ & $7.5 \pm 2.5$ & $296.3 \pm 30.7$ & $233.0 \pm 21.6$ & $63.3 \pm 13.0$ & $3.71 \pm 0.06$ & $1.64 \pm 0.26$ \\
\hline & $\begin{array}{l}\text { Cercidium } \\
\text { microphyllum }\end{array}$ & $0.77 \pm 0.04$ & $6.0 \pm 2.3$ & $7.4 \pm 2.7$ & $202.0 \pm 50.3$ & $155.4 \pm 32.7$ & $46.6 \pm 19.5$ & $3.49 \pm 0.21$ & $1.77 \pm 0.07$ \\
\hline & Encelia farinosa & $0.80 \pm 0.04$ & $13.4 \pm 9.8$ & $16.2 \pm 10.8$ & $226.1 \pm 50.9$ & $179.9 \pm 33.7$ & $46.2 \pm 18.6$ & $3.17 \pm 0.06$ & $1.99 \pm 0.0$ \\
\hline & Fouquieria diguetii & $0.80 \pm 0.02$ & $6.7 \pm 2.2$ & $7.8 \pm 2.5$ & $247.6 \pm 30.3$ & $196.5 \pm 18.5$ & $51.1 \pm 12.4$ & $2.97 \pm 0.02$ & $2.10 \pm 0.37$ \\
\hline & $\begin{array}{l}\text { Jatropha } \\
\text { cardiophylla }\end{array}$ & $0.85 \pm 0.01$ & $17.5 \pm 4.0$ & $21.2 \pm 4.5$ & $222.4 \pm 11.2$ & $188.9 \pm 8.3$ & $33.5 \pm 3.2$ & $3.30 \pm 0.25$ & $2.00 \pm 0.39$ \\
\hline & Larrea tridentata & $0.78 \pm 0.04$ & $4.1 \pm 2.3$ & $5.6 \pm 2.9$ & $272.2 \pm 42.8$ & $209.9 \pm 22.7$ & $62.2 \pm 20.4$ & $3.82 \pm 0.10$ & $1.30 \pm 0.20$ \\
\hline & Mimosa laxiflora & $0.81 \pm 0.04$ & $9.0 \pm 5.2$ & $11.6 \pm 6.3$ & $262.3 \pm 33.5$ & $210.7 \pm 18.6$ & $51.6 \pm 13.9$ & $3.04 \pm 0.27$ & $2.51 \pm 0.18$ \\
\hline & Olneya tesota & $0.76 \pm 0.03$ & $3.1 \pm 1.6$ & $4.3 \pm 1.9$ & $331.5 \pm 33.5$ & $251.9 \pm 16.8$ & $79.6 \pm 17.4$ & $3.01 \pm 0.09$ & $1.10 \pm 0.36$ \\
\hline
\end{tabular}


está diferencia no fue clara, lo cual sugiere la utilización de múltiples estrategias en las especies, que permiten asegurar una mayor ganancia de carbono al tiempo que la pérdida de agua no pone en riesgo su sobrevivencia.

El mejor ajuste en las relaciones $\mathrm{A}_{\text {area }}$ vs $\operatorname{Tr} \mathrm{y} \mathrm{A}_{\text {area }}$ vs gs para las especies en el sitio árido sugiere una mayor UEA cuando el agua está disponible (Figura 3). Es decir, hubo mayor fijación de carbono mediante la fotosíntesis con respecto a la pérdida de agua por transpiración, a través de una mayor sensibilidad de los estomas. Las especies desérticas exhiben atributos "xeromórficos" y bajas tasas de transpiración para evitar la pérdida sustancial de agua, que consecuentemente, provocan la disminución de la fotosíntesis (Seddon 1974). Una baja UEA puede ser atribuida a la alta demanda evaporativa del ambiente desértico (seco y cálido), por lo tanto, la sensibilidad en los estomas (Flexas et al. 2013, Gago et al. 2016) permite que cuando hay suficiente humedad en el suelo y la transpiración puede incrementar, la UEA disminuya durante ese período de tiempo, permitiendo aumentar rápidamente las tasas fotosintéticas y la ganancia de carbono (Seddon 1974).

Otras especies desérticas pueden mantener ganancias de carbono significativas como consecuencia de diferentes ajustes fisiológicos y no estructurales (Gibson \& Rundel 2012) o bien desacoplar sus requerimientos de humedad. Por ejemplo, algunas especies principalmente arbóreas freatofitas, obtienen el agua del subsuelo, y evitan el estrés hídrico atmosférico (Whitford 2002). En este sentido, la poca diferenciación de la UEA entre los sitios SJ y EC, pudo estar afectada por su desacoplamiento con la precipitación, dada la utilización de agua del subsuelo, como en el caso de $O$. tesota y $P$. velutina. Previamente se ha reportado la escasa relación entre la precipitación con la eficiencia en el uso del agua intrínseca $\left(\mathrm{UEA}_{\text {intrins }}=\mathrm{A}_{\text {area }} / \mathrm{gs}\right)$ en especies de Prosopis (Salazar et al. 2018), lo que indica el efecto que pueden tener la utilización del agua del subsuelo en las relaciones de UEA instantánea e intrínseca en sitios áridos y semiáridos. Las adecuaciones a escala local y no a las condiciones generalizadas de la precipitación, parecen ser más importantes y las mayores determinantes para el mantenimiento de una amplia diversidad de respuestas al uso del agua en las especies del sitio más árido (Tabla 3).

Efectos de la irradiancia y estrés lumínico en los atributos de las comunidades. Durante los eventos de precipitación en el desierto, las plantas activan su metabolismo y por ende la absorción de nutrientes y crecimiento. Por ello, en la comunidad semiárida, se esperaba una mayor limitación de nutrientes, así como una mayor eficiencia en su uso. Sin embargo, los resultados de este estudio indican que no hubo diferencias significativas en la eficiencia en el uso del agua $\left(\mathrm{UEA}_{\text {inst }}\right.$ o UEA $\left.\mathrm{Untrins}_{\text {int }}\right)$ o de nutrientes (EUN y EUP) entre el sitio árido (SJ) y semiárido (EC), dada la similitud en los promedios de las tasas fotosintéticas $\left(\mathrm{A}_{\text {area }}\right.$ y $\left.\mathrm{A}_{\text {mass }}\right)$, la gran variabilidad interespecífica en las variables hídricas y la alta concentración elemental de N y P. Por ejemplo, la concentración de $\mathrm{N}_{\text {mass }}$ en ambos sitios fue mayor que la media mundial de $18.9 \mathrm{mg} \mathrm{g}^{-1}$ (Tian et al. 2018), más alta
A)

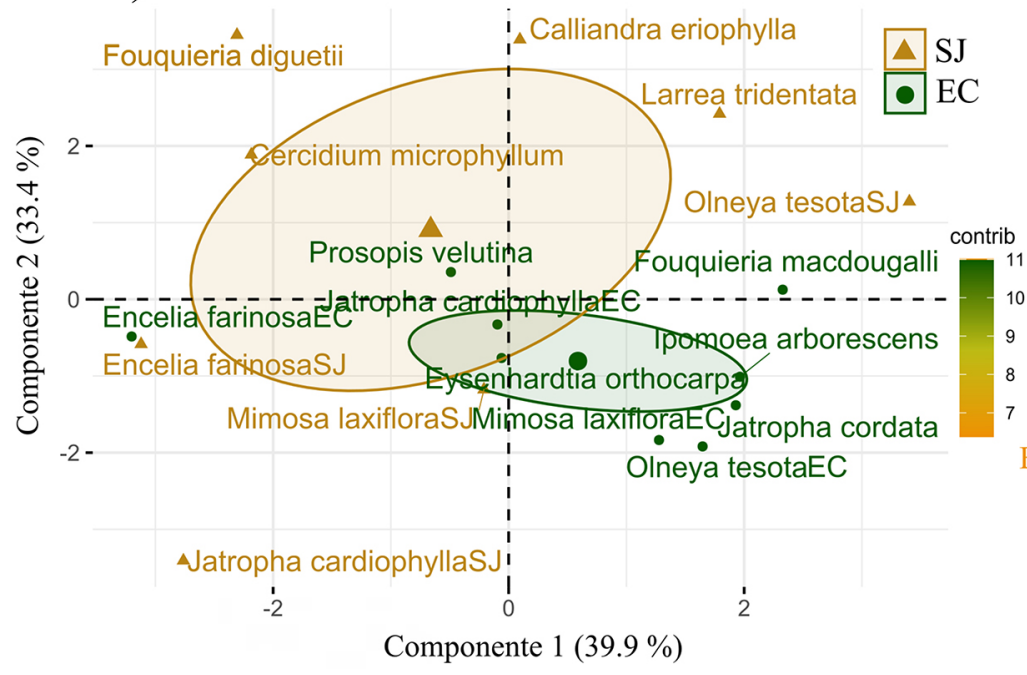

B)

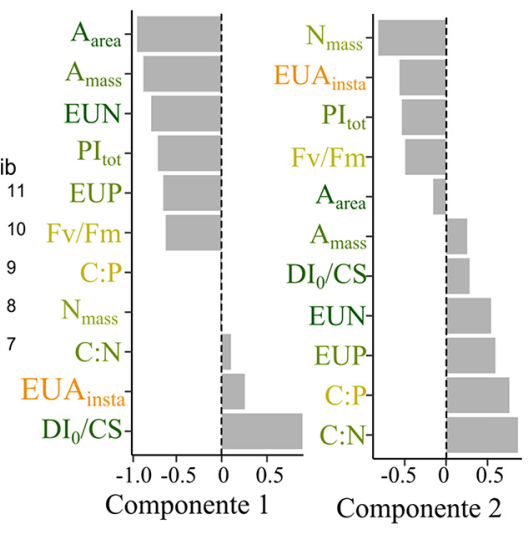

Figura 4. Análisis de componentes principales entre los sitios de San Judas (triángulo) y El Churi (círculo). Gráfica de vectores propios (A) y de cargas por componente (B). 
Tabla 5. Concentración de nutrientes foliares, proporciones estequiométricas y eficiencia en el uso de nutrientes en las especies de San Judas $\left(29^{\circ} 17^{\prime} 50.10^{\prime}\right.$ N y $\left.111^{\circ} 10^{\prime} 8.60^{\prime} \mathrm{W}\right)$ y El Churi $\left(28^{\circ} 42^{\prime} 6.77^{\prime} \mathrm{N}\right.$ y $110^{\circ} 32^{\prime} 15.64$ ” W). $\mathrm{N}_{\text {mass }}=$ nitrógeno foliar con base en masa $\left(\mathrm{mg} \mathrm{g}^{-1}\right), \mathrm{P}_{\text {mass }}=$ fósforo foliar con base en masa $\left(\mathrm{mg} \mathrm{g}^{-1}\right), \mathrm{N}_{\text {area }}=$ nitrógeno foliar con base en área $\left(\mathrm{g} \mathrm{m}^{-2}\right) \mathrm{C}: \mathrm{N}=$ cociente carbono/ nitrógeno, $\mathrm{N}: \mathrm{P}=$ cociente de nitrógeno/fósforo, $\mathrm{C}: \mathrm{P}=$ cociente carbono/fósforo, $\mathrm{EUN}=$ eficiencia en el uso del nitrógeno $\left(\mu \mathrm{mol} \mathrm{CO}_{2}\right.$ $\mathrm{mg}^{-1} \mathrm{~N} \mathrm{~s}^{-1}$ ) y EUP $=$ eficiencia en el uso del fósforo $\left(\mu \mathrm{mol} \mathrm{CO} \mathrm{mg}^{-1} \mathrm{P} \mathrm{s}^{-1}\right)$. Se presentan la media \pm desviación estándar. $\mathrm{n}=$ de 3 a 5 datos por especie. $\mathrm{NA}=$ No aplica.

\begin{tabular}{|c|c|c|c|c|c|c|c|c|c|}
\hline Sitio & Especie & $\mathbf{N}_{\text {mass }}$ & $\mathbf{P}_{\text {mass }}$ & $\mathbf{N}_{\text {area }}$ & $C: N$ & $\mathbf{N}: \mathbf{P}$ & $C: P$ & EUN & EUP \\
\hline \multirow[t]{9}{*}{ El Churi } & Encelia farinosa & $24.8 \pm 4.3$ & $2.3 \pm 0.5$ & $2.43 \pm 0.15$ & $17.2 \pm 2.53$ & $9.9 \pm 1.53$ & $183.8 \pm 38.5$ & 15.8 & 1.29 \\
\hline & Eysenhardtia orthocarpa & $33.9 \pm 3.9$ & $1.9 \pm \mathrm{NA}$ & $1.73 \pm 0.21$ & $13.3 \pm 1.53$ & $14.9 \pm \mathrm{NA}$ & $233.9 \pm \mathrm{NA}$ & 15.7 & 1.42 \\
\hline & Fouquieria macdougalii & $22.6 \pm 2.7$ & $1.9 \pm 0.1$ & $1.28 \pm 0.25$ & $19.4 \pm 2.38$ & $11.9 \pm 0.76$ & $231.8 \pm 13.8$ & 10.7 & 0.75 \\
\hline & Ipomoea arborescens & $32.4 \pm 3.6$ & $1.9 \pm 0.3$ & $1.49 \pm 0.27$ & $12.8 \pm 1.15$ & $16.3 \pm 2.32$ & $209.2 \pm 36.1$ & 10.2 & 0.65 \\
\hline & Jatropha cardiophylla & $26.7 \pm 2.9$ & $2.3 \pm 0.2$ & $1.65 \pm 0.30$ & $16.1 \pm 1.63$ & $11.5 \pm 2.79$ & $183.2 \pm 19.2$ & 12.2 & 1.05 \\
\hline & Jatropha cordata & $28.6 \pm 3.3$ & $2.4 \pm \mathrm{NA}$ & $1.59 \pm 0.37$ & $15.2 \pm 1.98$ & $8.9 \pm 2.07$ & $144.8 \pm 52.0$ & 8.0 & 0.41 \\
\hline & Mimosa laxiflora & $39.3 \pm 4.6$ & $1.9 \pm \mathrm{NA}$ & $2.41 \pm 0.26$ & $11.0 \pm 1.34$ & $21.8 \pm \mathrm{NA}$ & $224.3 \pm \mathrm{NA}$ & 7.4 & 1.21 \\
\hline & Olneya tesota & $31.7 \pm 5.1$ & $1.9 \pm 0.4$ & $2.16 \pm 0.31$ & $13.8 \pm 2.13$ & $16.0 \pm 1.21$ & $222.2 \pm 41.1$ & 9.0 & 0.81 \\
\hline & Prosopis velutina & $34.6 \pm 1.4$ & $1.3 \pm 0.0$ & $4.20 \pm 0.62$ & $13.8 \pm \mathrm{NA}$ & $26.9 \pm \mathrm{NA}$ & $371.5 \pm \mathrm{NA}$ & 5.9 & 1.6 \\
\hline \multirow[t]{8}{*}{ San Judas } & Calliandra eriophylla & $17.1 \pm 3.3$ & $0.88 \pm 0.1$ & $1.47 \pm 0.27$ & $25.4 \pm 4.39$ & $19.7 \pm 0.93$ & $500.7 \pm 78.3$ & 15.5 & 2.14 \\
\hline & Cercidium microphyllum & $27.9 \pm 2.5$ & $1.4 \pm 0.1$ & $2.70 \pm 0.21$ & $16.1 \pm 1.30$ & $18.8 \pm 0.74$ & $315.3 \pm 4.6$ & 14.7 & 2.07 \\
\hline & Encelia farinosa & $23.9 \pm 4.1$ & $2.5 \pm 0.8$ & $1.95 \pm 0.17$ & $14.9 \pm 1.08$ & $9.6 \pm 1.39$ & $142.0 \pm 10.3$ & 16.9 & 1.5 \\
\hline & Fouquieria diguetii & $17.5 \pm 1.1$ & $1.1 \pm 0.1$ & $1.59 \pm 0.05$ & $24.2 \pm 1.42$ & $16.3 \pm 2.38$ & $392.8 \pm 34.5$ & 21.5 & 2.54 \\
\hline & Jatropha cardiophylla & $37.77 \pm 4.8$ & $2.2 \pm 0.2$ & $1.97 \pm 0.15$ & $11.8 \pm 1.48$ & $17.3 \pm 0.88$ & $203.9 \pm 18.4$ & 10.4 & 1.41 \\
\hline & Larrea tridentata & $17.2 \pm 2.7$ & $1.4 \pm 0.3$ & $2.25 \pm 0.58$ & $27.1 \pm 4.50$ & $12.0 \pm 0.95$ & $328.0 \pm 79.7$ & 5.2 & 0.79 \\
\hline & Mimosa laxiflora & $31.8 \pm 3.9$ & $1.5 \pm 0.4$ & $1.97 \pm 0.18$ & $13.6 \pm 1.71$ & $20.7 \pm 2.09$ & $292.9 \pm 72.7$ & 9.6 & 1.43 \\
\hline & Olneya tesota & $22.9 \pm 3.9$ & $1.7 \pm 0.4$ & $1.65 \pm 0.42$ & $19.4 \pm 3.73$ & $14.3 \pm 5.39$ & $264.8 \pm 61.2$ & 6.5 & 0.66 \\
\hline
\end{tabular}

que la media de otras regiones áridas $\left(18.8 \pm 4.9 \mathrm{mg} \mathrm{g}^{-1} \mathrm{en}\right.$ China, Zhang et al. 2018) y similar a lo reportado para la zona de estudio $23.6 \pm 0.5 \mathrm{mg} \mathrm{g}^{-1}$ (Castellanos et al. 2018). Así, los resultados de este estudio son una evidencia más del alto contenido de $\mathrm{N}$ reportado para especies desérticas, en comparación con especies de otros biomas (Wright et al. 2001, Castellanos et al. 2018, Hinojo-Hinojo et al. 2018). De la misma manera, la concentración promedio de $\mathrm{P}_{\text {mass }}$ en ambos sitios, pero particularmente en el sitio semiárido (EC), fue significativamente mayor que la media mundial de $1.2 \mathrm{mg} \mathrm{g}^{-1}$ (Tian et al. 2018), y similar a las reportadas por Zhang et al. (2018) en China y Castellanos et al. (2018) en la misma región del estudio.

Aún a pesar del elevado promedio en la concentración de $\mathrm{P}$, los cocientes estequiométricos revelaron una posible limitación de $\mathrm{P}$ en la mayoría de las especies del estudio y en particular en el sitio árido (SJ), debido tal vez al mayor requerimiento de este elemento en procesos diferentes a la captación de $\mathrm{CO}_{2}$ en la fotosíntesis. Algunos estudios han relacionado el cociente $\mathrm{N}: \mathrm{P}$ con características del crecimiento en las especies y reportan que valores de $\mathrm{N}: \mathrm{P}<14$ se asocian con tasas mayores de crecimiento $\mathrm{y}$ limitación de $\mathrm{N}$ (47\% de las especies en este estudio), mientras que el crecimiento es menor cuando $\mathrm{N}: \mathrm{P}>16$ (53\% de las especies en este estudio) debido a la limitación de P en las hojas (Koerselman \& Meuleman 1996, Aerts \& Chapin 2000), aunque este porcentaje subió al $62.5 \%$ de las especies presentes en el sitio más árido. Los datos estequiométricos sugieren que, aun cuando no hubo diferencias significativas entre sitios, existe una tendencia 

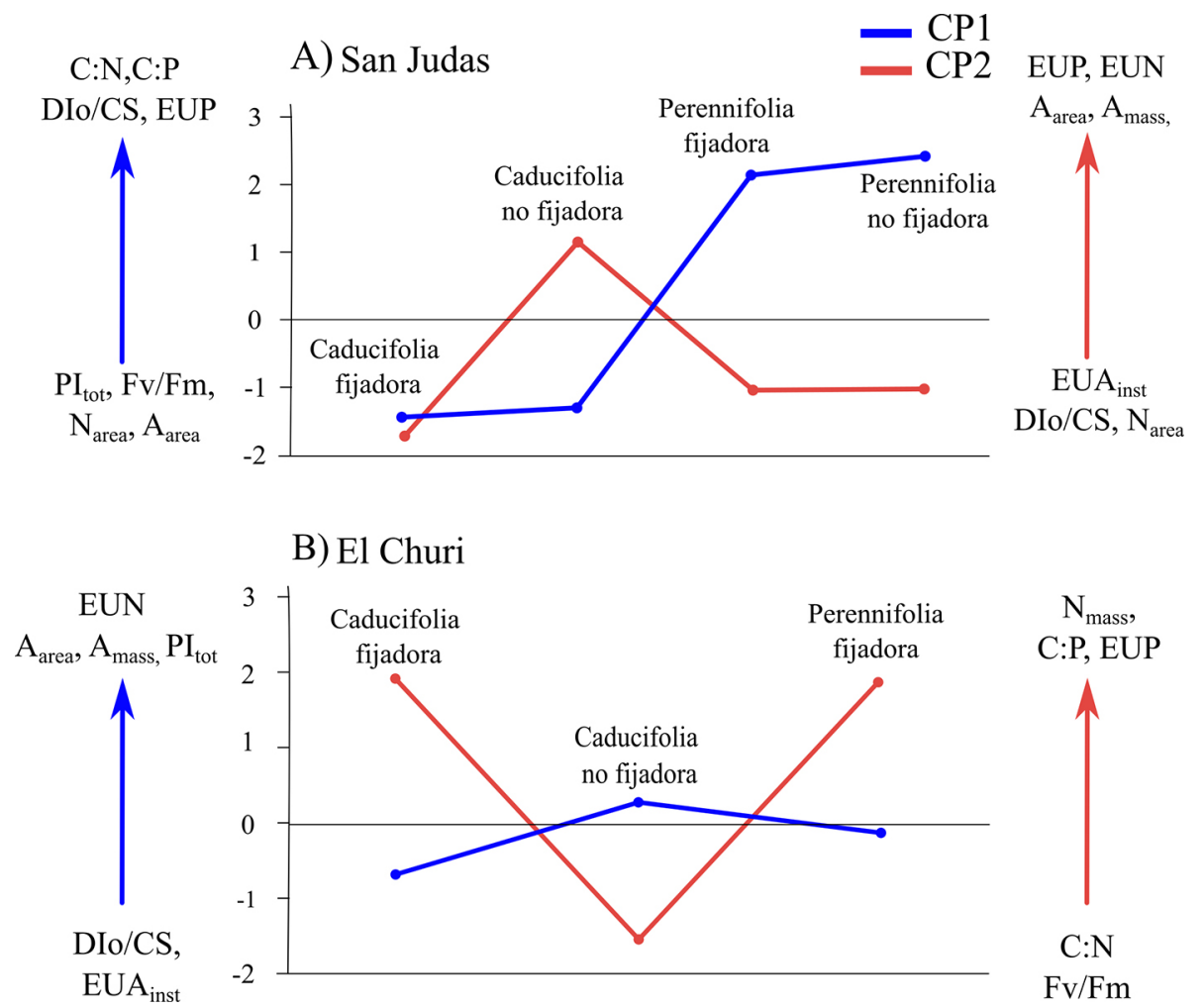

Figura 5. Cargas promedio de PCA obtenidas por tipo funcional para a) la comunidad de SJ y b) EC. CP1 = componente principal $1, \mathrm{CP} 2=$ componente principal 2.

a menor limitación por $\mathrm{P}$ en el sitio semiárido, como lo muestra la tendencia a la mayor EUP. El promedio del cociente N:P en ambos sitios, similar a lo reportado para la región (16.8 \pm 0.73 ; Castellanos et al. 2018) y mayor que la media reportada por Zhang et al. (2018) de $12.3 \pm 5.3 \mathrm{en}$ China, revela una amplia presencia de especies operando bajo limitación de $\mathrm{P}$ en los atributos y procesos ecofisiológicos en la región y una baja eficiencia en su uso, en particular en el sitio con mayor aridez.

En contraste, el cociente estequiométrico $\mathrm{C}: \mathrm{N}$ se ha asociado a estrategias para maximizar la ganancia de $\mathrm{C}$ mediante la fotosíntesis (Wright et al. 2004). Las tasas de fotosíntesis se relacionan con la concentración de $\mathrm{N}$ en las hojas (Field et al. 1983, Evans 1987), pero también con el uso eficiente de la luz en ambientes con alta intensidad lumínica (Sterner \& Elser 2002) y la mayor inversión en los complejos cosechadores de luz (Evans 1987, Ögren et al. 1993). En este estudio, no hubo relación entre $\mathrm{N}_{\text {area }}$ o $\mathrm{N}_{\text {mass }}$ con la fotosíntesis. Sin embargo, dadas las condiciones de alta irradiación y los múltiples mecanismos de fotoprotección que poseen las plantas cuando la fotosíntesis disminuye por condiciones de estrés (hídrico, lumínico o nutrimental), como los mecanismos relacionados con la fotorrespiración, la respiración y la disipación del exceso de radiación (Demmig-Adams \& Adams 2006), es esperable encontrar cocientes de $\mathrm{C}: \mathrm{N}$ más altos y menores eficiencias de uso de nitrógeno en relación con la fotosíntesis. En consecuencia, en este estudio, los mayores cocientes estequiométricos $\mathrm{C}: \mathrm{N}$ y $\mathrm{N}: \mathrm{P}$ parecen reflejar en ambos casos, estrategias de uso de $\mathrm{N}$ y P relacionadas más con la fotoprotección en las hojas, que en lograr mayores tasas fotosintéticas.

Lo mismo señalan las fuertes relaciones encontradas entre los atributos fotoquímicos (Fv/Fm y DIo/CS) y fotosintéticos que reflejan la importancia de los procesos relacionados con la fotoprotección en las estrategias de las especies y comunidades estudiadas (Figura 3 , Tabla 4). Por ejemplo, la relación inversa entre la fotosíntesis neta y tasa de disipación señala que la fotoprotección aumenta al tiempo que las tasas fotosintéticas disminuyen. Los mecanismos de disipación requieren de la inversión en pigmentos relacionados con la absorción de fotones, pero de manera significativa en pigmentos que permitan su disipación no fotoquímica cuando la fotosíntesis disminuye (Björkman \& Demmig-Adams 1995, Demmig-Adams \& Adams 2006). Esto sugiere que 
Tabla 6. Medias y diferencias de los atributos ecofisiológicos y estequiométricos entre las comunidades de San Judas (29 $17^{\prime} 50.10^{\prime \prime} \mathrm{N}$ y $111^{\circ} 10^{\prime} 8.60^{\prime}$ 'W) y El Churi (28 $42^{\prime} 6.77^{\prime}$ ' N y $110^{\circ} 32^{\prime} 15.64^{\prime}$ W). Se presentan media \pm desviación estándar, mediana, coeficiente de variación $(\mathrm{CV})$ y significancia estadística de la prueba. Diferencias significativas entre sitios en negritas.

\begin{tabular}{|c|c|c|c|c|c|c|c|}
\hline \multirow[b]{2}{*}{ Atributo } & \multicolumn{3}{|c|}{ San Judas } & \multicolumn{3}{|c|}{ El Churi } & \multirow[b]{2}{*}{ Significancia estadística } \\
\hline & $\operatorname{Media} \pm$ SD & Mediana & CV \% & Media \pm SD & Mediana & $\mathrm{CV} \%$ & \\
\hline $\mathrm{A}_{\text {area }}$ & $21.9 \pm 10.2$ & 20.5 & 46 & $20.1 \pm 7.0$ & 18.1 & 35 & - \\
\hline $\mathrm{A}_{\text {mass }}$ & $0.18 \pm 0.08$ & 0.15 & 48 & $0.14 \pm 0.084$ & 0.12 & 60 & - \\
\hline gs & $0.24 \pm 0.18$ & 1.37 & 78 & $0.21 \pm 0.152$ & 1.83 & 72 & - \\
\hline $\operatorname{Tr}$ & $6.71 \pm 3.7$ & 5.5 & 54 & $4.78 \pm 2.7$ & 5.7 & 56 & - \\
\hline$\varphi$ & $0.055 \pm 0.005$ & 0.054 & 10 & $0.051 \pm 0.004$ & 0.051 & 17 & - \\
\hline PLC & $63.7 \pm 32.7$ & 64.24 & 51 & $44.9 \pm 24.7$ & 38.8 & 62 & - \\
\hline PSL & $690.5 \pm 282.5$ & 621.3 & 39 & $709.5 \pm 547.3$ & 621.3 & 80 & - \\
\hline Ro & $3.82 \pm 1.69$ & 3.9 & 44 & $2.53 \pm 2.77$ & 1.7 & 113 & - \\
\hline $\mathrm{V}_{\mathrm{cmax} 25}$ & $102.1 \pm 46.4$ & 91.6 & 45 & $86.8 \pm 14.1$ & 89.8 & 16 & - \\
\hline $\mathrm{J}_{\max 25}$ & $152.6 \pm 134.9$ & 116 & 88 & $155.1 \pm 90.3$ & 130.9 & 58 & - \\
\hline $\mathrm{UEA}_{\text {inst }}$ & $3.9 \pm 2.1$ & 3.3 & 54 & $6.0 \pm 4.8$ & 4.1 & 80 & - \\
\hline $\mathrm{UEA}_{\text {intrins }}$ & $121.64 \pm 68.9$ & 104.8 & 56 & $151.0 \pm 103.6$ & 95.45 & 68 & \\
\hline EUN & $12.4 \pm 4.8$ & 12 & 38 & $10.7 \pm 3.6$ & 9.34 & 34 & - \\
\hline EUP & $1.02 \pm 0.4$ & 1.05 & 41 & $1.56 \pm 0.7$ & 1.46 & 38 & - \\
\hline Car tot & $1.76 \pm 0.5$ & 1.81 & 27 & $1.92 \pm 0.6$ & 1.82 & 30 & - \\
\hline Chl $a / b$ & $3.32 \pm 0.3 *$ & 3.2 & 9 & $3.0 \pm 0.4$ & 3 & 13 & $t=2.62, \mathrm{df}=46, P=0.01$ \\
\hline Chl tot & $26.7 \pm 8.7$ & 28.4 & 32 & $29.4 \pm 10.0$ & 29.25 & 34 & - \\
\hline AFE & $13.2 \pm 3.5$ & 12.3 & 26 & $17.4 \pm 4.1 * *$ & 18.8 & 23 & $t=-3.5, \mathrm{df}=47, P=0.001$ \\
\hline $\mathrm{Fv} / \mathrm{Fm}$ & $0.80 \pm 0.03$ & 0.8 & 48 & $0.79 \pm 0.05$ & 0.81 & 60 & - \\
\hline $\mathrm{PI}_{\mathrm{abs}}$ & $9.02 \pm 7.05$ & 6.8 & 78 & $6.78 \pm 4.0$ & 6.2 & 58 & - \\
\hline $\mathrm{PI}_{\text {tot }}$ & $11.21 \pm 8.04$ & 8.57 & 71 & $8.72 \pm 4.67$ & 8.37 & 53 & - \\
\hline RC/ABS & $0.71 \pm 0.15^{*}$ & 0.66 & 21 & $0.65 \pm 0.11$ & 0.66 & 16 & $t=-2.23, \mathrm{df}=74, P=0.028$ \\
\hline $\mathrm{RC} / \mathrm{CSo}$ & $174.8 \pm 31.6$ & 183.2 & 18 & $172.4 \pm 26.3$ & 172.9 & 15 & - \\
\hline $\mathrm{ABS} / \mathrm{CS}$ & $251.2 \pm 51.37$ & 240 & 20 & $268.4 \pm 45.25 *$ & 260 & 16 & $t=32.84, \mathrm{df}=45, P=0.00$ \\
\hline TRo/CS & $198.9 \pm 34.9$ & 192.7 & 17 & $211.4 \pm 27.7 *$ & 212.2 & 13 & $t=2.10, \mathrm{df}=75, P=0.038$ \\
\hline $\mathrm{DIo} / \mathrm{CS}$ & $52.3 \pm 18.9$ & 48.4 & 36 & $56.9 \pm 22.6$ & 49.32 & 39 & - \\
\hline $\mathrm{ETo} / \mathrm{CS}$ & $137.1 \pm 31.6$ & 138.5 & 14 & $144.0 \pm 26.3$ & 144.9 & 13 & - \\
\hline $\mathbf{N}_{\text {mass }}$ & $25.2 \pm 7.9$ & 25.5 & 31 & $30.6 \pm 5.9 *$ & 29.6 & 19 & $t=-2.59, \mathrm{df}=36, P=0.01$ \\
\hline $\mathrm{P}_{\text {mass }}$ & $1.6 \pm 0.60$ & 1.4 & 37 & $2.1 \pm 0.3 *$ & 1.9 & 28 & $t=-2.66, \mathrm{df}=29, P=0.01$ \\
\hline $\mathrm{C}: \mathrm{N}$ & $18.9 \pm 5.9 *$ & 16.9 & 31 & $14.7 \pm 2.8$ & 14.3 & 19 & $Z=-2.17, P=0.02$ \\
\hline C:P & $307.1 \pm 118.7 *$ & 312.1 & 38 & $212.4 \pm 56.0$ & 211 & 26 & $t=-2.97, \mathrm{df}=31, P=0.005$ \\
\hline $\mathrm{N}: \mathrm{P}$ & $16.3 \pm 4.1$ & 18 & 25 & $14.4 \pm 4.9$ & 14.3 & 34 & - \\
\hline
\end{tabular}

$t$ es el estadístico de la prueba de $t$ de Student para dos medias con una distribución normal, $Z$ es el estadístico utilizado para la prueba de diferencias de dos medias de Wilcoxon con distribución no normal. 
la mayor concentración de $\mathrm{Chl} a / b$ en el sitio con mayor aridez, podría ayudar a evitar el daño por estrés y alta irradiancia (Thayer \& Björkman 1990, Zhu et al. 2003), más cuando existe menor disponibilidad de N (Maina \& Wang 2015), tal como se observó en el sitio más árido (SJ). Aunque la inversión en fotoprotección puede ser costosa para la planta, parece evitar costos mayores a largo plazo ocasionados por fotodaño (Kothari et al. 2019, Marozas et al. 2019). Aun cuando las mediciones realizadas en este estudio recuperaron tasas instantáneas, es claro por los atributos mencionados, que las estrategias de las especies aquí estudiadas privilegian la ganancia de $\mathrm{C}$ en el largo plazo.

Atributos de las especies de las comunidades. Los atributos de las especies mostraron la gran diversidad de estrategias ecofisiológicas y estequiométricas relacionadas con la mayor conservación y no la eficiencia en el uso de recursos en ambas comunidades (Figura 4). Igual sucedió al explicar la estructuración de las comunidades con base en los principales tipos funcionales reconocidos (Figura 5). En el sitio árido (SJ), los atributos mostraron que las especies perennifolias, tanto fijadoras de nitrógeno $(\mathrm{NFj})$ como no fijadoras, se asociaron con atributos fotoprotectores en el primer componente, definido por mayores valores de C:P, C:N, DIo/CS y UEA inst $_{\text {, }}$ y menos con atributos relacionados con las tasas fotosintéticas instantáneas, evidenciando así las estrategias de conservación de recursos de este grupo funcional (Ackerly et al. 2002, Warren \& Adams 2004, Wright et al. 2004, Soh et al. 2019). El tipo funcional de las caducifolias fijadoras tuvo un comportamiento opuesto y privilegió los componentes instantáneos de fotosíntesis máxima $\left(\mathrm{A}_{\text {area }}\right)$, de $\mathrm{N}_{\text {area }}$ foliar y una mayor UEA $_{\text {inst }}$, lo que sugiere una estrategia explotativa (Adams et al. 2016, Wright et al. 2004). El cociente C:P concuerda con lo reportado para el grupo funcional de fijadoras de $\mathrm{N}$ en otros estudios (Adams et al. 2016, Castellanos et al. 2018), el cual se ha relacionado con el alto costo de energía y de fósforo requerido para el mantenimiento de los nódulos fijadores de nitrógeno (Lea \& Morot-Gaudry 2001, Delgado-Baquerizo et al. 2017, Smith et al. 1999).

En el sitio semiárido (EC) en cambio, los tipos funcionales de fijadoras de nitrógeno, tanto caducifolias como perennifolias, se asociaron de manera similar en sus atributos con la mayor UEA inst $, \mathrm{N}_{\text {mass }}, \mathrm{C}:$ P y EUP, y en menor grado DIo/CS (Figura 5). La relación del nitrógeno foliar $\left(\mathrm{N}_{\text {mass }}\right)$ y UEA $\mathrm{inst}_{\text {int }}$ refuerza lo planteado en estudios previos que indican que las altas concentraciones de $\mathrm{N}$ en las hojas de especies bajo condiciones de estrés hídrico moderado, permiten la mayor eficiencia en el uso del agua al permitir que los estomas moderadamente cerrados puedan mantener tasas fotosintéticas elevadas (Buckley et al. 2017, Field et al. 1983, Flexas \& Carriquí 2020), tal como ha sido reportado para las especies en el mismo sitio de estudio (Hinojo-Hinojo et al. 2018), aunque no se puede descartar la posibilidad de una mayor utilización de compuestos nitrogenados como osmolitos, aportando una ventaja competitiva en zonas secas (Erskine et al. 1996, Nolan et al. 2018), o su uso en funciones de fotoprotección. En general, las especies tanto en SJ como en EC, mostraron gran diversidad de estrategias de eficiencia en el uso de los recursos (agua y nutrientes) y mecanismos de fotoprotección, por lo que es evidente que, los atributos ecofisiológicos y estequiométricos estudiados, ayudan a explicar la dominancia y el éxito de especies y tipos funcionales de plantas con altas concentraciones de $\mathrm{N}$ en sus hojas y que están presentes en las comunidades del Desierto Sonorense y posiblemente en otras regiones áridas del país.

A nivel comunidad se concluye que la aridez afectó especialmente a los atributos relacionados con la composición elemental de $\mathrm{N}$ y $\mathrm{P}$, estequiométricos y algunos fotoquímicos. Contrario a lo esperado, en promedio, las especies y tipos funcionales de la comunidad árida se relacionaron más con las proporciones estequiométricas y eficiencia en el uso de nutrientes. Mientras que, la comunidad semiárida se asoció más a altos contenidos de nitrógeno y procesos de fotoquímica, como absorción de fotones y disipación. Las propiedades fotosintéticas y la eficiencia en el uso del agua tuvieron altas variaciones dentro de las comunidades, sugiriendo una gran diversidad de estrategias ecofisiológicas de las especies y los tipos funcionales presentes. Algunos estudios recientemente han sugerido que independientemente de las condiciones de estrés hídrico, otros factores selectivos locales promueven la mayor diversidad de estrategias de respuesta en las especies de una comunidad (Medeiros \& Drezner 2012, Bruelheide et al. 2018). Los tipos funcionales manifestaron en sus atributos, no solo concordancia con características afines al espectro económico de la hoja particularmente en las caducifolias y perennifolias, en específico fijadoras de $\mathrm{N}$, sino también y de manera más importante, con estrategias de fotoprotección a corto plazo, que resultan en condiciones de optimización de la ganancia de $\mathrm{C}$ en el largo plazo.

\section{Agradecimientos}

DMAA agradece el apoyo a CONACYT y Posgrado en Biociencias por la beca durante sus estudios de Maestría. AECV agradece el apoyo de CONACYT (CB61865 y 
CB223525) y TAMU-CONACYT (2016-011). AECV, JRLL y JMLIS agradecen a la Universidad de Sonora por su continuo apoyo. Agradecemos a JC Rodríguez, K. Campos, E. Martínez y el Laboratorio de Ecofisiología Vegetal por su apoyo en campo y laboratorio y a los Srs. Luis Sierra Maldonado (Rancho el Churi) y Alejandro Morales Aguilar (Rancho San Judas) por permitirnos el uso de sus instalaciones para realizar este trabajo de investigación.

\section{Literatura citada}

Ackerly DD, Knight CA, Weiss SB, Barton K, Starmer KP. 2002. Leaf size, specific leaf area and microhabitat distribution of chaparral woody plants: Contrasting patterns in species level and community level analyses. Oecologia 130: 449-457. DOI: https://doi.org/10.1007/ $\underline{\mathrm{s} 004420100805}$

Acuña-Acosta DM. 2018. Relación de la fluorescencia de la clorofila con estequiometría de $C, N$ y $P$ en hojas de especies y tipos funcionales del Desierto Sonorense. BScThesis. Universidad de Sonora.

Adams MA, Turnbull TL, Sprent JI, Buchmann N. 2016. Legumes are different: Leaf nitrogen, photosynthesis, and water use efficiency. Proceedings of the National Academy of Sciences of the United States of America 113: 4098-4103. DOI: https://doi.org/10.1073/ pnas. 1523936113

Aerts R, Chapin FS. 2000. The mineral nutrition of wild plants revisited: A re-evaluation. Advances in Ecological Research 30: 55. DOI: https://doi.org/10.1016/ S0065-2504(08)60016-1

Ahrar M, Doneva D, Tattini M, Brunetti C, Gori A, Rodeghiero M, Wohlfahrt G, Biasioli F, Varotto C, Loreto F, Velikova V. 2017. Phenotypic differences determine drought stress responses in ecotypes of Arundo donax adapted to different environments. Journal of Experimental Botany 68: 2439-2451. DOI: https://doi. org/10.1093/jxb/erx125

Andrews M, Raven JA, Lea PJ. 2013. Do plants need nitrate? The mechanisms by which nitrogen form affects plants. Annals of Applied Biology 163: 174-199. DOI: http://doi.org/10.1111/aab.12045

Arnon DI. 1949. Copper enzymes in isolated chloroplasts. Polyphenoloxidase in Beta vulgaris. Encyclopedia of Ecology 24: 1-14. DOI: https://doi.org/10.1104/ pp.24.1.1

Austin AT. 2011. Has water limited our imagination for aridland biogeochemistry. Trends in Ecology and Evo- lution 26: 229-235. DOI: https://doi.org/10.1016/j. tree.2011.02.003

Ávila-Lovera E, Haro R, Ezcurra E, Santiago LS. 2019. Costs and benefits of photosynthetic stems in desert species from southern California. Functional Plant Biology 46: 175-186. DOI: https://doi.org/10.1071/ FP18203

Baker NR. 2008. Chlorophyll fluorescence: A probe of photosynthesis in vivo. Annual Review of Plant Biology 59: 89-113. DOI: https://10.1146/annurev.arplant.59.032607.092759

Ball JT, Woodrow IE, Berry JA. 1987. A model predicting stomatal conductance and its contribution to the control of photosynthesis under different environmental conditions. In: Biggins J, ed. Progress in Photosynthesis Research, Dordrecht: Springer, pp 221-224. ISBN: 90 24734533

Bernacchi CJ, Singsaas EL, Pimentel C, Portis Jr AR, Long SP. 2001. Improved temperature response functions for models of Rubisco-limited photosynthesis. Plant, Cell and Environment 24: 253-259. DOI: https:// doi.org/10.1111/j.1365-3040.2001.00668.x

Björkman O, Demmig-Adams B. 1995. Regulation of photosynthetic light energy capture, conversion, and dissipation in leaves of higher plants. In: Schulze E-D, Caldwell MM eds, Ecophysiology of Photosynthesis, Berlin: Springer, pp 17-47. DOI: https://doi. org/10.1007/978-3-642-79354-7_2

Bruelheide H, Dengler J, Purschke O, Lenoir J, Jiménez-Alfaro B, Hennekens SM, Botta-Dukát Z, Chytrý M, Field R, Jansen F, Kattge J, Pillar VD, Schrodt F, Mahecha MD, Peet RK, Sandel B, van Bodegom P, Altman J, Alvarez-Dávila E, Arfin Khan MAS, Attorre F, Aubin I, Baraloto C, Barroso JG, Bauters M, Bergmeier E, Biurrun I, Bjorkman AD, Blonder B, Čarni A, Cayuela L, Černý T, Cornelissen JHC, Craven D, Dainese M, Derroire G, De Sanctis M, Díaz S, Doležal J, Farfan-Rios W, Feldpausch TR, Fenton NJ, Garnier E, Guerin GR, Gutiérrez AG, Haider S, Hattab T, Henry G, Hérault B, Higuchi P, Hölzel N, Homeier J, Jentsch A, Jürgens N, Kącki Z, Karger DN, Kessler M, Kleyer M, Knollová I, Korolyuk AY, Kühn I, Laughlin DC, Lens F, Loos J, Louault F, Lyubenova MI, Malhi Y, Marcenò C, Mencuccini M, Müller JV, Munzinger J, Myers-Smith IH, Neill DA, Niinemets Ü, Orwin KH, Ozinga WA, Penuelas J, Pérez-Haase A, Petř́k P, Phillips OL, Pärtel M, Reich PB, Römermann C, Rodrigues AV, Sabatini FM, Sardans J, Schmidt M, Seidler G, Silva Espejo JE, Silveira M, 
Smyth A, Sporbert M, Svenning JC, Tang Z, Thomas R, Tsiripidis I, Vassilev K, Violle C, Virtanen R, Weiher E, Welk E, Wesche K, Winter M, Wirth C, Jandt U. 2018. Global trait-environment relationships of plant communities. Nature Ecology and Evolution 2:1906 -1917. DOI: https://doi.org/10.1038/s41559018-0699-8

Buckley TN, Sack L, Farquhar GD. 2017. Optimal plant water economy. Plant Cell Environ 40: 881-896. DOI: https://doi.org/10.1111/pce.12823

Castellanos AE, Llano-Sotelo JM, Machado-Encinas LI, López-Piña JE, Romo-León JR, Sardans J, Peñuelas J. 2018. Foliar C, N, and P stoichiometry characterize successful plant ecological strategies in the Sonoran Desert. Plant Ecology 219: 775-788. DOI: https://doi. org/10.1007/s11258-018-0833-3

Castellanos-Villegas AE, Bravo LC, Koch GW, Llano J, López D, Méndez R, Rodríguez JC, Romo R, Sisk TD, Yanes G. 2010. Impactos ecológicos por el uso del terreno en el funcionamiento de ecosistemas áridos y semiáridos. In: FE Molina-Freaner, Van Devender TR, eds. Diversidad Biológica de Sonora. México: Comisión Nacional para el Conocimiento y Uso de la Biodiversidad - Universidad Nacional Autónoma de México, 157-186 pp. ISBN: 978-607-02-0427-2

Celaya-Michel H, Castellanos-Villegas AE. 2011. Mineralización de nitrógeno en el suelo de zonas áridas y semiáridas. Terra Latinoamericana 29: 343-356

Chabot B, Chabot J, Hicks D. 1982. The ecology of leaf life span. Annual Review of Ecology and Systematics 13: 229-259. DOI: https://doi.org/10.1146/annurev. es.13.110182.001305

Ciompi S, Gentili E, Guidi L, Soldatini GF. 1996. The effect of nitrogen deficiency on leaf gas exchange and chlorophyll fluorescence parameters in sunflower. Plant Science 118: 177-184. DOI: https://doi. org/10.1016/0168-9452(96)04442-1

Cowan IR, Farquhar GD. 1977. Stomatal function in relation to leaf metabolism and environment. Symposia of the Society for Experimental Biology 31: 471-505.

Delgado-Baquerizo M, Eldridge DJ, Maestre FT, Ochoa V, Gozalo B, Reich PB, Singh BK. 2017. Aridity Decouples C:N:P Stoichiometry Across Multiple Trophic Levels in Terrestrial Ecosystems. Ecosystems 21: 459-468. DOI: https://doi.org/10.1007/s10021-017$\underline{0161-9}$

Demmig-Adams B, Adams WW. 2006. Photoprotection in an ecological context: The remarkable complexity of thermal energy dissipation. New
Phytologist 172: 11-21. DOI: https://doi.org/10.1111/ j.1469-8137.2006.01835.x

Díaz S, Cabido M. 1997. Plant functional types and ecosystem function in relation to global change. Journal of Vegetation Science 8: 463-474. DOI: https://doi. org $/ 10.2307 / 3237198$

Duursma RA. 2015. Plantecophys - An R package for analysing and modelling leaf gas exchange data. PLoS ONE 10: 1-13. DOI: https://doi.org/10.1371/journal. pone. 0143346

Erskine PD, Stewart GR, Schmidt S, Turnbull MH, Unkovich M, Pate JS. 1996. Water availability - A physiological constraint on nitrate utilization in plants of Australian semi-arid mulga woodlands. Plant, Cell and Environment 19: 1149-1159. DOI: https://doi. org/10.1111/j.1365-3040.1996.tb00430.x

Evans J, Terashima I. 1987. Effects of Nitrogen Nutrition on Electron Transport Components and Photosynthesis in Spinach. Functional Plant Biology 14: 59. DOI: https://doi.org/10.1071/pp9870059

Evans J. 1987. The Relationship Between Electron Transport Components and Photosynthetic Capacity in Pea Leaves Grown at Different Irradiances. Functional Plant Biology 14: 157. DOI: https://doi.org/10.1071/ pp9870157

Ezcurra E. 2006. Natural history and evolution of the world deserts. In: Ezcurra E, ed. Global Deserts Outlook. Nairobi: United Nations Environment Programme (UNEP). pp. 1-26. ISBN: 9789280727227

Farquhar GD, von Caemmerer S, Berry JA. 1980. A biochemical-model of photosynthetic $\mathrm{CO}_{2}$ assimilation in leaves of $\mathrm{C}_{3}$ species. Planta 149: 78-90. DOI: https:// doi.org/10.1007/BF00386231

Fick SE, Hijmans RJ. 2017. Worldclim 2: New 1-km spatial resolution climate surfaces for global land areas. International Journal of Climatology 37: 4302-4315. DOI: https://doi.org/10.1002/joc.5086

Field C, Merino J, Mooney HA. 1983. Compromises between water-use efficiency and nitrogen-use efficiency in five species of California evergreens. Oecologia $\mathbf{6 0}$ : 384-389. DOI: https://doi.org/10.1007/BF00376856

Flexas J, Medrano H. 2002. Drought-inhibition of photosynthesis in $\mathrm{C}_{3}$ plants: Stomatal and non-stomatal limitations revisited. Annals of Botany 89: 183-189. DOI: https://doi.org/10.1093/aob/mcf027

Flexas J, Niinemets U, Gallé A, Barbour MM, Centritto M, Diaz-Espejo A, Douthe C, Galmés J, Ribas-Carbo M, Rodriguez PL, Rosselló F, Soolanayakanahally R, Tomas M, Wright IJ, Farquhar GD, Medrano H. 2013. 
Diffusional conductances to $\mathrm{CO}_{2}$ as a target for increasing photosynthesis and photosynthetic water-use efficiency. Photosynthesis Research 117: 45-59. DOI: https://doi.org/10.1007/s11120-013-9844-z

Flexas J, Carriquí M. 2020. Photosynthesis and photosynthetic efficiencies along the terrestrial plant's phylogeny: Lessons for improving crop photosynthesis. The Plant Journal 101: 964-978. DOI: https://doi. org/10.1111/tpj.14651

Franklin KA, Sommers PN, Aslan CE, López BR, Bronstein JL, Bustamante E, Búrquez A, Medellín RA, Marazzi B. 2016. Plant biotic interactions in the Sonoran Desert: Current knowledge and future research perspectives. International Journal of Plant Sciences 177: 217-234. DOI: https://doi.org/10.1086/684261

Gago J, Daloso DDM, Figueroa CM, Flexas J, Fernie AR. 2016. Relationships of leaf net photosynthesis, stomatal conductance and mesophyll conductance to primary metabolism: a multispecies metaanalysis approach. Plant Physiology 171: 265-279. DOI: https:// doi.org/10.1104/pp.15.01660

Gago J, Douthe C, Florez-Sarasam I, Escalona JM, Galmes J, Fernie AR, Flexas J, Medrano H. 2014. Opportunities for improving leaf water use efficiency under climate change conditions. Plant Science 226: 108-119. DOI: https://doi.org/10.1016/j.plantsci.2014.04.007

García-Moya E, McKell C. 1970. Contribution of shrubs to the nitrogen economy of a desert-wash plant community. Ecology 51: 81-88. DOI: https://doi. org $/ 10.2307 / 1933601$

Gibson AC, Rundel PW. 2012. Ecophysiology of photosynthesis in desert ecosystems. In: Flexas J, Loreto F, Medrano H, eds. Terrestrial Photosynthesis in a Changing Environment: A Molecular, Physiological, and Ecological Approach. Cambridge: Cambridge University Press. 450-451 pp. ISBN: 9781139051477

Granados-Sánchez D, López-Ríos F, Gama-Flores L. 1998. Adaptaciones y estrategias de las plantas de zonas áridas. Revista Chapingo Serie Ciencias Forestales $y$ del Ambiente 4: 169-178.

Guerra-Cantú J, Moreno-Limón S, Martínez-Rodríguez A, Gámez-González H, Núñez-González M, Moreno-Buentello OM. 2016. Determinación de pigmentos en orégano (Poliomintha bustamanta B. L. Turner.) en dos condiciones de crecimiento. Investigación y Desarrollo En Ciencia y Tecnología de Alimentos 1: 353-359.

Heberling M. 2013. A practical guide to measuring leaf- level photosynthesis. https://sites.google.com/site/fridleylab/home/protocols/Heberling_PhotosynthesisProtocol_Feb2014.pdf (accessed January 23, 2020).

Hinojo-Hinojo C, Castellanos AE, Llano-Sotelo J, Peñuelas J, Vargas R, Romo-Leon JR. 2018. High Vcmax, Jmax and photosynthetic rates of Sonoran Desert species: Using nitrogen and specific leaf area traits as predictors in biochemical models. Journal of Arid Environments 156: 1-8. DOI: https://doi.org/10.1016/j. jaridenv.2018.04.006

Huxman TE, Snyder KA, Tissue D, Leffler AJ, Ogle K, Pockman WT, Sandquist DR, Potts DL, Schwinning S. 2004. Precipitation pulses and carbon fluxes in semiarid and arid ecosystems. Oecologia 141: 254-268. DOI: https://doi.org/10.1007/s00442-004-1682-4

INEGI [Instituto Nacional de Estadística y Geografía]. 2015. Guía para la interpretación de cartografía Uso del suelo y vegetación Escala 1:250 000 Serie V. Instituto Nacional de Estadística y Geografía. México.

Ito A, Inatomi M. 2012. Water-use efficiency of the terrestrial biosphere: A model analysis focusing on interactions between the global carbon and water cycles. Journal of Hydrometeorology 13: 681-694. DOI: https:// doi.org/10.1175/JHM-D-10-05034.1

Kassambara A, Mundt F. 2020. Factoextra: Extract and visualize the results of multivariate data analyses. https:// rpkgs.datanovia.com/factoextra/index.html (accessed July 23, 2020).

Kattge J, Diaz S, Lavorel S, Prentice IC, Leadley P, Bönisch G, Garnier E, Westoby M, Reich PB, Wright, Cornelissen JHC, Violle C, Harrison SP, Van Bodegom PM, Reichstein M, Enquist BJ, Soudzilovskaia NA, Ackerly DD, Anand M, Atkin O, Bahn M, Baker TR, Baldocchi D, Bekker R, Blanco CC, Blonder $\mathrm{B}$, Bond WJ, Bradstock R, Bunker DE, Casanoves F, Cavender-Bares J, Chambers JQ, Chapin III FS, Chave J, Coomes D, Cornwell WK, Craine JM, Dobrin B, Duarte L, Durka W, Elser J, Esser G, Estiarte M, Fagan WF, Fang J, Fernández-Méndez F, Fidelis A, Finegan B, Flores O, Ford H, Frank D, Freschet GT, Fyllas NM, Gallagher RV, Green WA, Gutierrez AG, Hickler T, Higgins SI, Hodgson JG, Jalili A, Jansen S, Joly CA, Kerkhoff AJ, Kirkup D, Kitajima K, Kleyer M, Klotz S, Knops JMH, Kramer K, Kühn I, Kurokawa H, Laughlin D, Lee TD, Leishman M, Lens $\mathrm{F}$, Lenz T, Lewis SL, Lloyd J, Llusià J, Louault F, Ma S, Mahecha MD, Manning P, Massad T, Medlyn BE, Messier J, Moles AT, Müller SC, Nadrowski K, Naeem S, Niinemets Ü, Nöllert S, Nüske A, Ogaya 
R, Oleksyn J, Onipchenko Vg, Onoda Y, Ordoñez J, Overbeck G, Ozinga Wa, Patiño S, Paula S, Pausas JG, Peñuelas J, Phillips OL, Pillar V, Poorter H, Poorter L, Poschlod P, Prinzing A, Proulx R, Rammig A, Reinsch S, Reu B, Sack L, Salgado-Negret B, Sardans J, Shiodera S, Shipley B, Siefert A, Sosinski E, Soussana JF, Swaine E, Swenson N, Thompson K, Thornton P, Waldram M, Weiher E, White M, White S, Wright SJ, Yguel B, Zaehle S, Zanne AE, Wirth C. 2011. Try-a global database of plant traits. Global Change Biology 17: 2905-2935. DOI: https://doi. org/10.1111/j.1365-2486.2011.02451.x

Koerselman W, Meuleman AFM. 1996. The Vegetation N:P Ratio: A New Tool to Detect the Nature of Nutrient Limitation. The Journal of Applied Ecology 33: 1441. DOI: https://doi.org/10.2307/2404783

Köhler IH, Macdonald AJ, Schnyder H. 2016. Last-century increases in intrinsic water-use efficiency of grassland communities have occurred over a wide range of vegetation composition, nutrient inputs, and soil pH. Plant Physiology 170: 881-890. DOI: https://doi. org/10.1104/pp.15.01472

Kothari S, Montgomery R, Cavender-Bares J. 2019. Throwing shade: Physiological responses to light explain competition and facilitation in a tree diversity experiment. BioRxiv 845701. DOI: https://doi. org $/ 10.1101 / 845701$

Laity J. 2008. Deserts and Desert Enviroment. Chichester: Wiley-Blackwell. ISBN: 9781444300741

Lea PJ, Morot-Gaudry JF. 2001. Plant Nitrogen. Berlin: Springer-Verlag. pp 407. ISBN: 3540677992

Maina JN, Wang Q. 2015. Seasonal Response of Chlorophyll a/b Ratio to Stress in a Typical Desert Species: Haloxylon ammodendron. Arid Land Research and Management 29: 321-334. DOI: https://doi.org/10.108 0/15324982.2014.980588

Marozas V, Augustaitis A, Pivoras A, Baumgarten M, Mozgeris G, Sasnauskienè J, Dautartè A, Abraitienè $\mathrm{J}$, Bičenkienė S, Mordas G, Ulevičius V, Matyssek R. 2019. Comparative analyses of gas exchange characteristics and chlorophyll fluorescence of three dominant tree species during the vegetation season in hemi-boreal zone, Lithuania. Journal of Agricultural Meteorology 75: 3-12. DOI: https://doi.org/10.2480/ agrmet.D-18-00004

Medeiros AS, Drezner TD. 2012. Vegetation, climate, and soil relationships across the Sonoran Desert. Ecoscience 19: 1-13. DOI: https://doi.org/10.2980/19-2-3485 Murphy J, Riley J. 1962. A modified single solution meth- od for the determination of phosphate in natural waters. Analytica Chimica Acta 27: 31-36. DOI: https:// doi.org/10.1016/S0003-2670(00)88444-5

Nolan RH, Tarin T, Rumman R, Cleverly J, Fairweather KA, Zolfaghar S, Santini NS, O'Grady AP, Eamus D. 2018. Contrasting ecophysiology of two widespread arid zone tree species with differing access to water resources. Journal of Arid Environments 153: 1-10. DOI: https://doi.org/10.1016/j.jaridenv.2018.01.003

Noy-Meir I. 1973. Desert ecosystems: environment and producers. Annual review of ecology and systematics 4: 25-51. DOI: https://doi.org/10.1146/annurev. es.04.110173.000325

Ögren E, Jakobsen I, Evans JR. 1993. Photosynthetic light-response curves - 2. Gradients of light absorption and photosynthetic capacity. Planta 189: 191-200. DOI: https://doi.org/10.1007/BF00195076

Perez-Harguindeguy N, Díaz S, Garnier E, Lavorel S, Poorter H, Jaureguiberry P, Bret-Harte MS, Cornwell WK, Craine JM, Gurvich DE, Urcelay C, Veneklaas EJ, Reich PB, Poorter L, Wright IJ, Ray P, Enrico L, Pausas JG, de Vos AC, Buchmann N, Funes G, Quétier F, Hodgson JG, Thompson K, Morgan HD, ter Steege H, van der Heijden MGA, Sack L, Blonder B, Poschlod P, Vaieretti MV, Conti G, Staver AC, Aquino S, Cornelissen JHC. 2016. Corrigendum to: New handbook for standardized measurement of plant functional traits worldwide. Australian Journal of Botany 61: 167-234. DOI: https://doi.org/10.1071/ BT12225

Prăvălie R, Bandoc G, Patriche C, Sternberg T. 2019. Recent changes in global drylands: Evidences from two major aridity databases. Catena 178: 209-231. DOI: https://doi.org/10.1016/j.catena.2019.03.016

R Core Team. 2020. R: A language and environment for statistical computing. R Foundation for Statistical Computing, Vienna, Austria. URL: https://www.Rproject.org/

Reich P, Wright I, Cavender-Bares J, Craine J, Oleksyn J, Walters MB, Journal I, Reich PB, Westoby M, Walters M. 2003. The evolution of plant functional variation: traits, spectra, and strategies. International Journal of Plant Sciences 164: S143-S164. DOI: https://doi. org/10.1086/374368

Reich P. 2014. The world-wide "fast-slow" plant economics spectrum: A traits manifesto. Journal of Ecology 102: 275-301. DOI: https://doi.org/10.1111/1365$\underline{2745.12211}$

Rzedowski J. 2006. Vegetación de México. 1ra. Edición 
digital, Comisión Nacional para el Conocimiento y Uso de la Biodiversidad, México.

RStudio Team. 2020. RStudio: Integrated Development for R. RStudio, PBC, Boston, MA URL: http://www. rstudio.com/

Salazar PC, Navarro-Cerrillo RM, Cruz G, Villar R. 2018. Intraspecific leaf functional trait variability of eight Prosopis pallida tree populations along a climatic gradient of the dry forests of northern Peru. Journal of Arid Environments 152: 12-20. DOI: https://doi. org/10.1016/j.jaridenv.2018.01.010

Schneider CA, Rasband WS, Eliceiri KW. 2012. NIH Image to ImageJ: 25 years of image analysis. Nat Methods 9: 671-675. DOI: https://doi.org/10.1038/nmeth.2089

Seddon G. 1974. Xerophytes, xeromorphs and sclerophylls: the history of some concepts in ecology. Biological Journal of the Linnean Society 6: 65-87. DOI: https://doi.org/10.1111/j.1095-8312.1974.tb00714.x

Sharkey TD, Bernacchi CJ, Farquhar GD, Singsaas EL. 2007. Fitting photosynthetic carbon dioxide response curves for $\mathrm{C}_{3}$ leaves. Plant, Cell and Environment 30: 1035-1040. DOI: https://doi.org/10.1111/j.13653040.2007.01710.x

Skujins J. 1981. Nitrogen cycling in arid ecosystems. In: Clark FE, Rosswall, T, eds. Terrestrial Nitrogen Cycles. Processes, Ecosystem Strategies and Management Impacts. Sweden Stockholm: Swedish National Sciences Research Council. pp. 477-491. ISBN: 9154602904

Smith RJ, Lea PJ, Gallon JR. 1999. Nitrogen fixation. In: Lea P, Leegood R, eds. Plant Biochemistry and Molecular Biology, Chichester, U.K.: John Wiley y Sons. pp. 477-491. ISBN: 978-0-471-97683-7

Soh WK, Yiotis C, Murray M, Parnell A, Wright IJ, Spicer RA, Lawson T, Caballero R, McElwain JC. 2019. Rising $\mathrm{CO}_{2}$ drives divergence in water use efficiency of evergreen and deciduous plants. Science Advances 5: 1-11. DOI: https://doi.org/10.1126/sciadv.aax7906

Sterner RW, Elser JJ. 2002. Ecological Stoichiometry. The Biology of Elements from Molecules to the Biosphere. Nueva Jersey, USA: Princeton University. ISBN: 9780691074917

Strasser RJ, Srivastava A, Tsimilli-Michael M. 2000. The fluorescence transient as a tool to characterize and screen photosynthetic samples. In: Yunus M, Pathre U, Mohanty P. Probing Photosynthesis: Mechanisms, Regulation and Adaptation. London: Taylor and Francis, pp. 445-483. ISBN: 9780748408214

Taiz L, Zeiger E. 2006. Plant Physiology. Sinauer Associates Inc., Sunderland, 782 ISBN: 9780878938568
Thayer SS, Björkman O. 1990. Leaf Xanthophyll content and composition in sun and shade determined by HPLC. Photosynthesis Research 23: 331-343. DOI: https://doi.org/10.1007/BF00034864

Tian D, Yan Z, Niklas KJ, Han W, Kattge J, Reich PB, Luo Y, Chen Y, Tang Z, Hu H, Wright IJ, Schmid B, Fang J. 2018. Global leaf nitrogen and phosphorus stoichiometry and their scaling exponent. National Science Review 5: 728-739. DOI: https://doi.org/10.1093/ nsr/nwx142

Violle C, Navas M-L, Vile D, Kazakou E, Fortunel C, Hummel I, Garnier E. 2007. Let the concept of trait be functional! Oikos 116: 882-892. DOI: https://doi. org/10.1111/j.2007.0030-1299.15559.x

Warren CR, Adams MA. 2004. Evergreen trees do not maximize instantaneous photosynthesis. Trends in Plant Science 9: 270-274. DOI: https://doi.org/10.1016/j. tplants.2004.04.004

West NE, Skujins J. 1978. Nitrogen in Desert Ecosystems. Stroudsburg: Dowden, Hutchinson and Ross. ISBN: 0879333332

Whitford WG. 2002. Ecology of Desert Systems. San Diego: Academic Press. ISBN: 9780127472614

Wright IJ, Reich PB, Westoby M, Ackerly DD, Baruch Z, Bongers F, Cavender-Bares J, Chapin T, Cornelissen JH, Diemer M, Flexas J, Garnier E, Groom PK, Gulias J, Hikosaka K, Lamont, T Lee, W Lee, C Lusk, JJ Midgley, ML Navas, Ü Niinemets, J Oleksyn, N Osada BB, Poot P, Prior L, Pyankov VI, Roumet C, Thomas SC, Tjoelker MG, Veneklaas EJ, Villar R. 2004. The worldwide leaf economics spectrum. $\mathrm{Na}$ ture 428: 821-827. DOI: https://doi.org/10.1038/nature02403

Wright IJ, Reich PB, Westoby M. 2001. Strategy-shifts in leaf physiology, structure, and nutrient content between species of high and low rainfall, and high and low nutrient habitats. Functional Ecology 15: 423-434. DOI: https://doi.org/10.1046/j.0269-8463.2001.00542.x

Yamori W. 2016. Photosynthetic response to fluctuating environments and photoprotective strategies under abiotic stress. Journal of Plant Research 129: 379-395. DOI: https://doi.org/10.1007/s10265-016-0816-1

Zhang X, Guan T, Zhou J, Cai W, Gao N, Du H, Jiang L, Lai L, Zheng Y. 2018. Community characteristics and leaf stoichiometric traits of desert ecosystems regulated by precipitation and soil in an arid area of China. International Journal of Environmental Research and Public Health 15: 109. DOI: https://doi.org/10.3390/ ijerph15010109 
Zhu XY, Wang SM, Zhang CL. 2003. Composition and characteristic differences in photosynthetic membranes of two ecotypes of reed (Phragmites communis L.). Photosynthetica 42: 97-104. DOI: https://doi. org/10.1023/A:1025820731410

Živčák M, Olšovská K, Slamka P, Galambošová J, Rataj

Editor de sección: Numa Pavón

Contribución de los autores: DMAA diseñó, ejecutó el trabajo de campo, analizó los datos y escribió el manuscrito; AECV diseñó y apoyó con trabajo de campo, análisis de datos y escritura del manuscrito; JMLIS asesoró y ejecutó el análisis de nutrientes y base de datos, y revisó el documento; JRRL asesoró y revisó el documento.
V, Shao HB, Kalaji HM, Brestič M. 2014. Measurements of chlorophyll fluorescence in different leaf positions may detect nitrogen deficiency in wheat. Zemdirbyste-Agriculture 101: 437-444. DOI: https://doi. org/10.13080/z-a.2014.101.056 